\title{
Decomposing and backtesting a flexible specification for CoVaR*
}

\author{
Giovanni Bonaccolto ${ }^{\dagger}$ \\ Massimiliano Caporin $\ddagger$
}

Sandra Paterlini ${ }^{\S}$

This version: June 24, 2019

\begin{abstract}
We introduce the Conditional Autoregressive Quantile-Located VaR (QL-CoCaViaR), that extends the Conditional Value-at-Risk (Adrian and Brunnermeier, 2016) by using an estimation process capturing the state of joint distress of the financial system and of individual companies. Furthermore, we include autoregressive components of conditional quantiles to explicitly model volatility clustering and heteroskedasticity. We support our model with a large empirical analysis, in which we use both classical and novel backtesting methods. Our results show that the quantilelocated relationships lead to relevant improvements in terms of predictive accuracy during stressed periods, providing a valuable tool for regulators to assess systemic events.
\end{abstract}

Keywords: CoVaR, quantile-location, backtest, decomposition

\section{Introduction}

Recent financial crises have highlighted the need to develop better tools and measures to quantify and predict systemic risk, emphasizing the importance of the interconnectedness of firms, their exposure to systemic events as well as the marginal effect each company has on the entire system. The literature provides more than one definition of systemic risk (see, e.g., Rosengren (2010), Billio et al. (2012) and

\footnotetext{
${ }^{*}$ We thank the anonymous reviewers, Marcelo Medeiros, Pawel Polak, Christian Hafner, Elena Dumitrestu, Alberto Plazzi, Angelo Ranaldo, Giovanni De Luca, Gianluca Cubadda, Giuseppe Storti, Alessandro Pollastri, as well as the participants to the Mathematical and Statistical Methods for Actuarial Sciences and Finance (MAF 2018) held in Madrid, the 11th Annual Meeting of the Society for Financial Econometrics (SoFiE) held in Lugano, the 49th Scientific meeting of the Italian Statistical Society held in Palermo, the Quantitative Finance and Financial Econometrics Conference held in Marseille, the 19th Workshop on Quantitative Finance held in Rome, the 42nd Annual Meeting of the Association for Mathematics Applied to Social and Economic Sciences (AMASES) held in Naples, for helpful comments and suggestions.

${ }^{\dagger}$ University of Enna "Kore", Italy. Email: giovanni.bonaccolto@unikore.it

${ }^{\ddagger}$ Department of Statistical Sciences, University of Padova, Italy. Email: massimiliano.caporin@unipd.it

${ }^{\S}$ Corresponding author. Department of Economics and Management, University of Trento, Italy. Department of Finance and Accounting, European Business School, Germany. Email: sandra.paterlini@unitn.it
} 
Acharya et al. (2017)), highlighting the complex nature of the financial system. This has prompted the development of several systemic risk measures, see Benoit et al. (2017) for a recent survey. The collapse of Lehman Brothers and the aftermath of the subprime crisis have prompted regulators to move beyond the unconditional Value-at-Risk (VaR), which does not capture the dependence structure of extreme co-movements in stock markets and the consequent spillover effects generated during stressed phases. Following this need, Adrian and Brunnermeier (2016) introduced the Conditional Value-at-Risk (CoVaR), that has become one of the most successful measures for systemic risk. In contrast to the VaR, which only quantifies the individual tail-risk of the system, the CoVaR takes into account the impact on the entire system of a company in distress. By relying on quantile regression, Adrian and Brunnermeier (2016) introduced a simple but yet informative risk measure. As a result, the CoVaR has attracted a relevant attention and has been applied in many empirical studies, see, among other, López-Espinosa et al. (2012), Bernal et al. (2014), Castro and Ferrari (2014), Bernardi et al. (2015), López-Espinosa et al. (2015), and Bernardi et al. (2017).

Here, we extend the CoVaR along two main directions. First, we take into account the fact that the volatility - and then the distribution - of financial returns changes over time. Nevertheless, the CoVaR method does not explicitly take into account the heteroskedastic behavior of financial returns. We fill this gap by adding autoregressive components capturing the quantiles' dynamics over time, inspired by the Conditional Autoregressive Value-at-Risk (CaViaR) introduced by Engle and Manganelli (2004). ${ }^{1}$ In particular, we analyze whether and in what measure the inclusion of the CaViaR's components - which capture the persistence of quantiles over time - affects the relations between the financial system and the conditioning distressed company. Second, the CoVaR's parameters are estimated conditional on the entire distribution of the returns of the distressed company. Nevertheless, correlations between financial institutions increase during stressed periods, when the risk of contagion threatens the stability of the entire economy due to potential spillover effects. Consequently, the information content of extreme returns, i.e. focusing on the left quantiles of distributions, becomes increasingly relevant when accentuating the role of distress in identifying financial connections. Here, we emphasize the impact exerted by a distressed company on the market by focusing on the tails of the conditioning company returns distribution. Therefore, we increase/better capture the distress degree in the relations between this company and the entire system by directly linking the left tails of their returns' distributions. In other words, we use an estimation process that reflects the state of joint distress of the system and of a company. We define this aspect as quantile-location.

By combining the dynamics in quantiles and the quantile-location we thus introduce the Quantile-Located Conditional Autoregressive Value-at-Risk (QL-CoCaViaR), where the conditional VaR depends on autoregressive components and is linked to a quantile of the independent (conditioning)

\footnotetext{
${ }^{1}$ Bekaert et al. (2015) used a different approach based on GARCH models with relevant insights in terms of market returns quantiles.
} 
variable. We estimate the QL-CoCaViaR's parameters with a weighted quantile regression model building on kernel-based weights, following the approach that Sim and Zhou (2015) used to study the relations between oil prices and stock returns. To the best of our knowledge, such a method has never been tested within a CoVaR framework for systemic risk. Note that the QL-CoCaViaR model nests the CoVaR specification as well as two additional sub-models, in which either quantile dynamics or quantile-location are absent (the QL-CoVaR and the CoCaViaR, respectively). Furthermore, we introduce an additional innovative element, that is, a decomposition of the resulting $\triangle \mathrm{QL}-\mathrm{CoCaViaR}$ into seven components having a specific economic interpretation (see Section 2.3). We also study the contribution of these components to the overall risk measure. Therefore, we can analyze how the CaViaR and the quantile-located effects interact when combined in the same model - the QL-CoCaViaR - and whether this combination outperforms the CoCaViaR and the QL-CoVaR estimated separately.

Other extensions of the CoVaR are available in the literature. The contributions that most closely relate to our are White et al. (2015) and Girardi and Ergun (2013). In particular, White et al. (2015) proposed the 'VAR for VaR' - a multivariate version of the CaViaR model introduced by Engle and Manganelli (2004). Similar to our approach, the 'VAR for VaR' captures both the persistence of the system's quantiles and the relations between the lower quantiles of the system and of the $i$-th company. Nevertheless, our approach differs in three important points. First, the 'VAR for VaR' links the past of a single company to the present of the system, whereas we focus on contemporary relationships to asses how the system immediately reacts when an institution is in distress. Second, in White et al. (2015), the quantiles of the system and of the conditioning company are estimated in a bivariate setting in which the covariates are their respective latent (lagged) values. In contrast, the return of the conditioning company that we include in the equation of the system's quantile is not latent, being observed. Third, in White et al. (2015), the conditioning company is in distress when its return is exactly at its VaR. Similar to Girardi and Ergun (2013), we provide more flexibility as the distress of the conditioning company does not necessarily occur when it is exactly at its VaR. In particular, in Girardi and Ergun (2013), the conditioning institution can be at most at its VaR to be in distress. In contrast, we are aware that the distress state of the conditioning company might not be confined to the case of observing returns lower than or equal to a given quantile. In fact, we consider the impact exerted by the distressed company on the market by focusing on the (left and right) neighborhood of its Value-at-Risk, increasing the flexibility and smoothing discontinuities in the identification of the company distress.

We compare the competing risk measures by using a large dataset including more than 1,000 banks and insurance companies. Starting from an in-sample analysis, we checked that the relations between the system and the individual companies become stronger when considering the quantile-located effects, that is, when accentuating the distress degree in their connections. In fact, the distance between the quantile-located measures $-\Delta \mathrm{QL}-\mathrm{CoVaR}$ and $\Delta \mathrm{QL}-\mathrm{CoCaViaR}$ - and the other indicators $-\Delta \mathrm{CoVaR}$ 
and $\Delta \mathrm{CoCaViaR}$ - is relatively low during calm periods and becomes accentuated during stressed phases - e.g., during the subprime crisis, during the 'internet bubble' in 2000, after the terrorist attacks in September 2001, around the stock market crash in 2002, during the war in Iraq (2001-2003) and during the European sovereign debt crisis (2010 - 2011). We then highlight the capability of the quantile-located measures- $\Delta \mathrm{QL}-\mathrm{CoVaR}$ and $\Delta \mathrm{QL}-\mathrm{CoCaViaR}$ - to react more strongly during events of system-wide relevance. In particular, the introduction of quantile-located effects does not simply provide a shift in the measured systemic impact but also accounts for changes in the structural relations between the individual companies and the market when the overall system is in turmoil.

We also evaluate the out-of-sample performance of the risk measures using various backtesting methods. Well-known backtesting approaches are, in our opinion, not appropriate. SANDRA: I would rather say that they are useful but do not consider relevant aspects for quantile-located risk measures. For instance, the standard coverage tests developed by Kupiec (1995) and Christoffersen (1998) or the loss functions used by Caporin (2008) do not take into account the impact of individual companies on the system. We then introduce novel backtesting methods tailored to the quantile-located risk measures we use here. Our analysis shows that the quantile-located relations are particularly useful in improving the predictive accuracy during stressed periods, with the QL-CoVaR outperforming the other measures. In addition to the tail coverage, we also evaluate the capability of the competing risk measures to identify ex-ante systemic important financial companies. Works comparing $\Delta$-type risk measures, possibly nested, such as the $\Delta \mathrm{CoVaR}$ or the $\Delta \mathrm{QL}-\mathrm{CoCaViaR}$, are not common in the literature. Indeed, to the best of our knowledge, there is no backtest in the literature specifically designed to evaluate and compare $\Delta$-type risk measures. Building on a sparse principal component analysis (Zou et al., 2006), we fill this gap by using a method that enables us to automatically identify the institutions that produce a significant impact on the financial system during periods of distress and to compare ex-ante estimates with ex-post realizations. In doing so, we check that the $\Delta \mathrm{QL}-\mathrm{CoCaViaR}$ provides more accurate results, that support the economic and statistical relevance of our method. This is mainly due to the introduction of quantile-located effects. Our proposal can thus be used by regulators as a signalling tool to either prevent or mitigate the effects of extreme events, with benefits in terms of financial stability. Finally, we also asses the systemic relevance of each company in our dataset and show that our method allows to capture the riskiness of the financial institutions that are classified as global systemically important by the Financial Stability Board, even when relying on a limited set of information.

The paper is structured as follows. We present the risk measures and the backtesting methods in Section 2. Section 3 describes the dataset and the empirical set-up, whereas Section 4 reports the empirical results. Section 5 concludes. 


\section{Notation and methods}

Let $y_{t}$ and $x_{i, t}$ be the returns of the financial system and of the $i$-th company at time $t$, respectively, for $i=1, \ldots, N$ and $t=1, \ldots, T . \mathbf{M}_{t}$ is a $k$-dimensional row vector including a set of control variables observed at time $t$. Let $Q_{\tau}\left(x_{i, t} \mid \mathbf{I}_{t-1}\right)$ be the $\tau$-th quantile of $x_{i, t}$, for $\tau \in(0,1)$, conditional to the information set $\mathbf{I}_{t-1}$, where $\mathbf{I}_{t-1}=\left(y_{t-1}, x_{i, t-1}, \mathbf{M}_{t-1}\right)$. In contrast, $Q_{\theta}\left(y_{t} \mid \mathbf{I}_{t-1}, x_{i, t}\right)$ is the $\theta$-th quantile of $y_{t}$ conditional to the information set available at $t-1$ as well as to the return of the $i$-th company observed at time $t$, for $\theta \in(0,1)$. For simplicity, we set $Q_{\tau}\left(x_{i, t} \mid \mathbf{I}_{t-1}\right) \equiv Q_{\tau}\left(x_{i, t}\right)$ and $Q_{\theta}\left(y_{t} \mid \mathbf{I}_{t-1}, x_{i, t}\right) \equiv$ $Q_{\theta}^{(i)}\left(y_{t}\right) . \quad \theta$ and $\tau$ take low values, typically in the interval $(0,0.05]$, as we focus on the left-tail dependence between $y_{t}$ and $x_{i, t}$. Hence, $Q_{\theta}^{(i)}\left(y_{t}\right)$ and $Q_{\tau}\left(x_{i, t}\right)$ are interpreted as the Values-at-Risk (VaRs) of the financial system and of the $i$-th company at the levels $\theta$ and $\tau$, respectively.

\subsection{Conditional Value--at-Risk}

Adrian and Brunnermeier (2016) introduced the Conditional Value-at-Risk (CoVaR), the estimation of which builds on the following (linear) conditional quantile models:

$$
\begin{gathered}
Q_{\tau}\left(x_{i, t}\right)=\alpha_{\tau}^{(i)}+\beta_{\tau}^{(i)} \mathbf{M}_{t-1}^{\prime}, \\
Q_{\theta}^{(i)}\left(y_{t}\right)=\delta_{\theta}^{(i)}+\lambda_{\theta}^{(i)} x_{i, t}+\gamma_{\theta}^{(i)} \mathbf{M}_{t-1}^{\prime},
\end{gathered}
$$

where $\alpha_{\tau}^{(i)}, \delta_{\theta}^{(i)}$, and $\lambda_{\theta}^{(i)}$ are scalars, whereas $\boldsymbol{\beta}_{\tau}^{(i)}$ and $\boldsymbol{\gamma}_{\theta}^{(i)}$ are $k$-dimensional row vectors of parameters. The subscripts of the parameters in (1) - (2) point out their dependence on the quantiles levels $\tau$ and $\theta$, respectively; in contrast, the superscript $(i)$ indicates that (1)-(2) are specific to the $i$-th company, for $i=1, \ldots, N$. Although the intercept $\delta_{\theta}^{(i)}$ and the vector $\gamma_{\theta}^{(i)}$ in $(2)$ are not directly linked to the $i$-th company, they might be affected by the relations between $y_{t}$ and $x_{i, t}$. Therefore, $\delta_{\theta}^{(i)}$ and $\gamma_{\theta}^{(i)}$ in (2) are also indexed by $(i)$. Note that $Q_{\tau}\left(x_{i, t}\right)$ and $Q_{\theta}^{(i)}\left(y_{t}\right)$ in $(1)-(2)$ depend on a common set of control variables, that is, the ones included in $\mathbf{M}_{t-1}^{\prime}$.

The parameters in (1) - (2) are estimated by using the quantile regression method introduced by Koenker and Bassett (1978). Their standard errors could be computed following various approaches. Here, we use a bootstrap method (Efron, 1979), that is, the xy-pair approach of Kocherginsky (2003) that provides accurate results without any distributional assumption. After obtaining the estimated quantile $\widehat{Q}_{\tau}\left(x_{i, \tau}\right)=\widehat{\alpha}_{\tau}^{(i)}+\widehat{\boldsymbol{\beta}}_{\tau}^{(i)} \mathbf{M}_{t-1}^{\prime}$, the CoVaR of the financial system, conditional to the VaR of the $i$-th company, is computed as follows:

$$
\operatorname{CoVaR}(i)=\widehat{\delta}_{\theta}^{(i)}+\widehat{\lambda}_{\theta}^{(i)} \widehat{Q}_{\tau}\left(x_{i, t}\right)+\widehat{\gamma}_{\theta}^{(i)} \mathbf{M}_{t-1}^{\prime}
$$

For the sake of brevity, we do not use $t, \theta$ and $\tau$ as subscripts, as well as $(i)$ as superscript, when 
we refer to the CoVaR as a risk measure throughout the paper. Hence, the CoVaR represents the risk of the financial system at the level $\theta$ (as in the case of the traditional VaR) conditional to the fact that the $i$-th company is in distress, that is, its return is equal to its $\operatorname{VaR}: x_{i, t}=\widehat{Q}_{\tau}\left(x_{i, \tau}\right)$. We can also compute the CoVaR conditional to the normal (or median) state of the $i$-th company:

$$
\operatorname{CoVaR} R_{t, \theta, 1 / 2}^{(i)}=\widehat{\delta}_{\theta}^{(i)}+\widehat{\lambda}_{\theta}^{(i)} \widehat{Q}_{1 / 2}\left(x_{i, t}\right)+\widehat{\gamma}_{\theta}^{(i)} \mathbf{M}_{t-1}^{\prime}
$$

We highlight the fact that the quantile level $\theta$ does not change from (3) to (4). Indeed, the coefficients in (3) - (4) are identical, being estimated from the same quantile regression model in (2). By subtracting $\operatorname{CoVaR}(i)$ from $C o V a R_{t, \theta, \tau}^{(i)}$, we compute the so-called $\Delta \mathrm{CoVaR}$ (Adrian and Brunnermeier, 2016) to quantify the marginal contribution of the $i$-th company to the systemic risk. Given that $\operatorname{CoVa} R_{t, \theta, 1 / 2}^{(i)}$ is always parameterized with respect to the median state of the $i$-th company, we can omit the level $1 / 2$ as subscript of the $\Delta$ CoVaR measure as follows:

$$
\Delta \operatorname{CoVaR}(i)=\operatorname{CoV} a R_{t, \theta, \tau}^{(i)}-\operatorname{CoVaR} R_{t, \theta, 1 / 2}^{(i)}=\widehat{\lambda}_{\theta}^{(i)}\left[\widehat{Q}_{\tau}\left(x_{i, t}\right)-\widehat{Q}_{1 / 2}\left(x_{i, t}\right)\right]
$$

For simplicity, we estimate the quantiles of $y_{t}$ and $x_{i, t}-\widehat{Q}_{\theta}^{(i)}\left(y_{t}\right)$ and $\widehat{Q}_{\tau}\left(x_{i, t}\right)$ - at the same level, that is, $\theta=\tau$. Hence, we can further simplify the notation by setting $\Delta \operatorname{CoVaR} R_{t, \theta, \tau}^{(i)} \equiv \Delta \operatorname{CoVa} R_{t, \tau}^{(i)}$.

\subsection{Quantile-Located Conditional Autoregressive Value-at-Risk}

We generalize the CoVaR along two directions: i) estimating the model when the $i$-th company is in distress; and ii) accounting for the well-known presence of heteroskedasticity in financial returns. Starting from the first point, Adrian and Brunnermeier (2016) estimate the CoVaR's parameters along the full support of the returns' distribution of the $i$-th company. Therefore, the link between the VaR of the system and the VaR of the $i$-th company consists in plugging $\widehat{Q}_{\tau}\left(x_{i, t}\right)$, estimated from (1), into (2), obtaining the CoVaR in (3). Nevertheless, the parameters in (2), and then the coefficients in (3), are functions of $\theta$ only - they link the covariates to the $\theta$-th quantile of $y_{t}$-without considering the role of $\tau$, that is, the reference quantile for the conditioning financial institution. In other words, the estimation process behind (3) depends on $x_{i, t}$ and not on $Q_{\tau}\left(x_{i, t}\right)$. As a result, the observations in the support of $x_{i, t}$ are equally weighted.

However, measures of extreme risk quantify the losses occurring during tail events, such as financial crises. In such periods, the correlations among financial institutions increase and the risk of contagion threatens the stability of the entire economy, due to potential spillover effects. Consequently, the information content of extreme risk measures becomes increasingly relevant when accentuating the distress degree in financial connections. In particular, in the context of the CoVaR proposed by Adrian and Brunnermeier (2016), we can increase the distress degree by linking the left tails of the distributions 
of $y_{t}$ and $x_{i, t}$. Indeed, we estimate (2) by assuming that the financial system and the $i$-th company are simultaneously in the left tails of their distributions. Therefore, we restrict the attention on the impact exerted by $x_{i, t}$ on $\widehat{Q}_{\theta}^{(i)}\left(y_{t}\right)$, when the former is in the neighborhood of its $\tau$-th quantile. This allows for a further degree of flexibility, as the parameters monitoring the impact of $x_{i, t}$ on $y_{t}$ might depend on the location of both $x_{i, t}$ and $y_{t}$ along their marginal support. Borrowing from Sim and Zhou (2015), we resort to a weighted quantile regression model, with kernel-based weights, to estimate the relations between the quantiles of $y_{t}$ and $x_{i, t}$. We define this generalization as quantile-location. Our approach corresponds to a nonparametric quantile regression, in which the knots used to obtain local estimates are fixed at specific conditional quantiles of $x_{i, t}$ (Koenker, 2005).

The second axis along which we generalize the CoVaR deals with the volatility clustering of financial time series (Cont, 2001). Volatility changes over time, affecting the distribution and, thus, the quantiles of assets' returns. The latter are thus dynamic, but the CoVaR (Adrian and Brunnermeier, 2016) neglects such a phenomenon. Indeed, (1) - (2) do not include any factor reflecting the heteroskedastic behavior of returns and, thus, the dynamics of both $Q_{\theta}^{(i)}\left(y_{t}\right)$ and $Q_{\tau}\left(x_{i, t}\right)$. Here, we fill this gap by including autoregressive components that capture the quantiles' dynamics, inspired by the Conditional Autoregressive Value-at-Risk (CaViaR) introduced by Engle and Manganelli (2004). ${ }^{2}$

We define the model that resulted from the two extensions described above as Quantile-Located Conditional Autoregressive Value-at-Risk (QL-CoCaViaR). The QL-CoCaViaR has the following form:

$$
\begin{gathered}
Q_{\tau}\left(x_{i, t}\right)=\alpha_{\tau}^{(i)}+\phi_{1, \tau}^{(i)} Q_{\tau}\left(x_{i, t-1}\right)+\phi_{2, \tau}^{(i)} f\left(x_{i, t-1}\right)+\boldsymbol{\beta}_{\tau}^{(i)} \mathbf{M}_{t-1}^{\prime} \\
Q_{\theta, \tau}^{(i)}\left(y_{t}\right)=\delta_{\theta, \tau}^{(i)}+\psi_{1, \theta, \tau}^{(i)} Q_{\theta, \tau}^{(i)}\left(y_{t-1}\right)+\psi_{2, \theta, \tau}^{(i)} f\left(y_{t-1}\right)+\lambda_{\theta, \tau}^{(i)} x_{i, t}+\gamma_{\theta, \tau}^{(i)} \mathbf{M}_{t-1}^{\prime} .
\end{gathered}
$$

The model building on (6) - (7) has two main differences with respect to (1) - (2). First, the latent autoregressive components $Q_{\tau}\left(x_{i, t-1}\right)$ and $Q_{\theta}^{(i)}\left(y_{t-1}\right)$ smooth the changes in the estimated quantiles over time, capturing their dynamics. ${ }^{3}$ Moreover, $\phi_{2, \tau}^{(i)}$ and $\psi_{2, \theta, \tau}^{(i)}$ link $Q_{\tau}\left(x_{i, t}\right)$ and $Q_{\theta}^{(i)}\left(y_{t}\right)$ to the past of $x_{i, t}$ and $y_{t}$, respectively. We set $f\left(x_{i, t-1}\right)=\left|x_{i, t-1}\right|$ and $f\left(y_{t-1}\right)=\left|y_{t-1}\right|$, as suggested by Engle and Manganelli (2004). Such a choice implies a direct response of the quantiles to the processes of $x_{i, t}$ and $y_{t}$, symmetrically treating the effect of positive and negative returns. Second, the parameters in (7) have both $\theta$ and $\tau$ as subscripts, as they depend on the quantiles levels of both $y_{t}$ and $x_{i, t}$. In fact,

\footnotetext{
${ }^{2}$ The heteroskedasticity in the CoVaR's dynamics is also considered, under a different approach, based on GARCH modelling, by Girardi and Ergun (2013).

${ }^{3}$ Other works in the literature that study, under different viewpoints, quantile autoregressions are, for instance, Koenker and Xiao (2004) and Li et al. (2015).
} 
the unknown parameters in (7) are estimated from the following minimization problem:

$$
\begin{aligned}
\underset{\delta_{\theta, \tau}^{(i)}, \psi_{1, \theta, \tau}^{(i)}, \psi_{2, \theta, \tau}^{(i)}, \lambda_{\theta, \tau}^{(i)}, \gamma_{\theta, \tau}^{(i)}}{\arg \min } & \sum_{t=1}^{T} \rho_{\theta}\left[y_{t}-\delta_{\theta, \tau}^{(i)}-\psi_{1, \theta, \tau}^{(i)} Q_{\theta, \tau}^{(i)}\left(y_{t-1}\right)-\psi_{2, \theta, \tau}^{(i)}\left|y_{t-1}\right|-\lambda_{\theta, \tau}^{(i)} x_{i, t}-\gamma_{\theta, \tau}^{(i)} \mathbf{M}_{t-1}^{\prime}\right] \\
\times & K\left(\frac{\widehat{F}_{t \mid t-1}\left(x_{i, t}\right)-\tau}{h}\right)
\end{aligned}
$$

where $\rho_{\theta}(e)=e\left(\theta-\mathbf{1}_{\{e<0\}}\right)$ is the asymmetric loss function used in the quantile regression method by Koenker and Bassett (1978); $\mathbf{1}_{\{\cdot\}}$ is an indicator function, taking the value of 1 if the condition in $\{\cdot\}$ is true, the value of 0 otherwise; $K(\cdot)$ is a kernel function, with bandwidth $h$, whereas $\widehat{F}_{t \mid t-1}\left(x_{i, t}\right)$ is the probability level corresponding to the empirical conditional quantile of $x_{i, t}$.

We describe the procedure to estimate $\widehat{F}_{t \mid t-1}\left(x_{i, t}\right)$ below. We first estimate a large set of $x_{i, t}$ 's quantiles in the support $\tau \in(0,1)$ from the quantile regression model (6). Note that the values of $Q_{\tau}\left(x_{i, t}\right)$ estimated for $\tau \in(0,1)$ are no longer interpreted as Values-at-Risk of $x_{i, t}$, given that we consider the entire distribution of $x_{i, t}$, not only the left tail. When estimating multiple quantiles in the interval $(0,1)$, the standard method introduced by Koenker and Bassett (1978) does not guarantee their coherence, that is, their monotonicity for $\tau \in(0,1)$ - e.g., we might obtain $\widehat{Q}_{0.95}\left(x_{i, t}\right)<\widehat{Q}_{0.90}\left(x_{i, t}\right)$. In order to obtain a valid conditional distribution of $x_{i, t}$, we use the method developed by Bondell et al. (2010). This allows us to compute a large set of quantiles having a monotonic behavior for $\tau \in(0,1)$, that we linearly interpolate to obtain $\widehat{F}\left(x_{i, t} \mid \mathbf{M}_{t-1}^{\prime}\right)$ - the conditional distribution of $x_{i, t}$ at time $t$. Finally, we recover $\widehat{F}_{t \mid t-1}\left(x_{i, t}\right)$, that is, the probability level, extrapolated from $\widehat{F}\left(x_{i, t} \mid \mathbf{M}_{t-1}^{\prime}\right)$, corresponding to the realization $x_{i, t}$. On the basis of a rolling window procedure, we estimate $\widehat{F}_{t \mid t-1}\left(x_{i, t}\right)$ for $t=w s, w s+1, \ldots, T$, where $w s$ denotes the length of the estimation window. In general, larger values of $w s$ allow us to improve the statistical properties of the quantiles $\widehat{Q}_{\tau}\left(x_{i, t}\right)$, for $\tau \in(0,1)$, resulting then in more stable estimates. Besides, a larger set of estimated quantiles $\widehat{Q}_{\tau}\left(x_{i, t}\right)$ provides a more accurate conditional distribution $\widehat{F}\left(x_{i, t} \mid \mathbf{M}_{t-1}^{\prime}\right)$, reducing the errors coming from their interpolation. Nevertheless, increasing both the length of the estimation window and the number of quantiles would lead to sensibly higher costs in terms of computational burden.

When deriving $\widehat{F}_{t \mid t-1}\left(x_{i, t}\right)$, it is important to highlight that we differ from Sim and Zhou (2015) who, instead, rely on the unconditional (full sample) empirical quantile:

$$
\widehat{F}\left(x_{i, t}\right)=T^{-1} \sum_{k=1}^{T} \mathbf{1}_{\left\{x_{i, k}<x_{i, t}\right\}}
$$

in place of $\widehat{F}_{t \mid t-1}\left(x_{i, t}\right)$.

The use of (9) implicitly relies on the stability of quantiles over time. Therefore, it neglects the fact that financial returns are typically affected by heteroskedasticity and other elements that impact on 
the location, scale and symmetry of their distributions. ${ }^{4}$ In contrast, $\widehat{F}_{t \mid t-1}\left(x_{i, t}\right)$ builds on a dynamic conditional distribution of $x_{i, t}$. As a result, $\widehat{F}_{t \mid t-1}\left(x_{i, t}\right)$ captures the heteroskedastic behavior and, in general, the instability of the $x_{i, t}$ 's distribution over time.

We estimate the parameters in (8) and their standard errors by using a modified version of the algorithm proposed by Engle and Manganelli (2004) for the CaViaR. This was needed to take into account the smoothing effect of the kernel function $K(\cdot)$. Furthermore, the estimation process in (8) relies on the kernel bandwidth value $h$ : the smaller $h$, the smaller the bias of the estimates, but the larger their variance, and vice versa. In contrast to Sim and Zhou (2015), which report results for a bandwidth value equal to 0.05 , we test the sensitivity of the estimates for different $h$ values, as a robustness check. We then check whether, and in what measure, the results change according to the choice of the bandwidth value $h$. After estimating the parameters in (7) and setting $\theta=\tau$, we compute the QL-CoCaViaR at the $\tau$-th level as follows:

$$
Q L-\operatorname{CoCaViaR} R_{t, \theta, \tau}^{(i)}=\widehat{\delta}_{\theta, \tau}^{(i)}+\widehat{\psi}_{1, \theta, \tau}^{(i)} \widehat{Q}_{\theta, \tau}^{(i)}\left(y_{t-1}\right)+\widehat{\psi}_{2, \theta, \tau}^{(i)}\left|y_{t-1}\right|+\widehat{\lambda}_{\theta, \tau}^{(i)} \widehat{Q}_{\tau}\left(x_{i, t}\right)+\widehat{\gamma}_{\theta, \tau}^{(i)} \mathbf{M}_{t-1}^{\prime}
$$

where $\widehat{Q}_{\tau}\left(x_{i, t}\right)=\widehat{\alpha}_{\tau}^{(i)}+\widehat{\phi}_{1, \tau}^{(i)} \widehat{Q}_{\tau}\left(x_{i, t-1}\right)+\widehat{\phi}_{2, \tau}^{(i)}\left|x_{i, t-1}\right|+\widehat{\boldsymbol{\beta}}_{\tau}^{(i)} \mathbf{M}_{t-1}^{\prime}$.

We also estimate the QL-CoCaViaR at the median state of the $i$-th company ( $Q L-C o C a V i a R_{t, \theta, 1 / 2}^{(i)}$ ), that is, by replacing $\tau$ with $1 / 2$ in (6) - (8). The evaluation of the impact exerted by a financial company in distress on the system, as in the original CoVaR framework, becomes now quite complex due to the joint presence of autoregressive and quantile-located components. Then, Subsection 2.3 provides directions for disentangling and interpreting the outcomes of the resulting $\Delta \mathrm{QL}-\mathrm{CoCaViaR}$. We close this subsection by noticing that our model includes two sub-models: the QL-CoVaR and the CoCaViaR. These two cases correspond to either the absence of quantiles' dynamics (QL-CoVaR) or the absence of quantile-location (CoCaViaR). The corresponding models' equations simplify accordingly. As for the estimation process, we can use the same approach adopted for the QL-CoCaViaR when focusing on the QL-CoVaR. In contrast, when estimating the CoCaViaR, we can follow the estimation approach proposed by Engle and Manganelli (2004) for the CaViaR.

\subsection{Interpreting the $\Delta \mathrm{QL}-\mathrm{CoCaViaR}$ estimates}

The complex structure of the $\Delta \mathrm{QL}-\mathrm{CoCaViaR}$ might challenge the financial interpretation of the model's estimates. We show how the model provides a rich and financially relevant interpretation about the link between a conditioning company in distress and the financial system using a companion

\footnotetext{
${ }^{4}$ This evidence is confirmed in our dataset by using the $D Q$ and the $S Q$ tests developed by $\mathrm{Qu}(2008)$. The $D Q$ test checks for the presence of structural breaks in the conditional distribution of a given variable. In contrast, the $S Q$ test verifies the presence of structural breaks at specific quantiles levels (in our analysis, we test the $5 \%, 50 \%$ and $95 \%$ levels). Both the $D Q$ and the $S Q$ provide evidence against the assumption of stable quantiles and distributions over time. Therefore, we prefer not to use $F_{T}\left(x_{i, t}\right)$ defined in (9) because it does not capture such dynamics. The output of both the $D Q$ and the $S Q$ tests is available upon request.
} 
representation. Given $\theta=\tau$, the $\Delta \mathrm{QL}-\mathrm{CoCaViaR}$ can be rewritten as follows: ${ }^{5}$

$$
\begin{aligned}
\Delta Q L-C o C a V i a R_{t, \tau}^{(i)} & =\underbrace{Q L-C o C a V i a R_{t, \theta, \tau}^{(i)}-Q L-C o C a V i a R_{t, \theta, 1 / 2}^{(i)}}_{c_{1, \tau}^{(i)}} \\
& =\underbrace{\left(\widehat{\delta}_{\theta, \tau}^{(i)}-\widehat{\delta}_{\theta, 1 / 2}^{(i)}\right)}_{c_{3, t, \tau}^{(i)}}+\underbrace{\widehat{\lambda}_{\theta, \tau}^{(i)}\left[\widehat{Q}_{\tau}\left(x_{i, t}\right)-\widehat{Q}_{1 / 2}\left(x_{i, t}\right)\right]}_{\theta, \tau} \\
& +\underbrace{\left(\widehat{\lambda}_{\theta, \tau}^{(i)}-\widehat{\lambda}_{\theta, 1 / 2}^{(i)}\right) \widehat{Q}_{1 / 2}\left(x_{i, t}\right)}_{c_{2, t, \tau}^{(i)}}+\underbrace{\left(\widehat{\gamma}_{\theta, \tau}^{(i)}-\widehat{\gamma}_{\theta, 1 / 2}^{(i)}\right) \mathbf{M}_{t-1}^{\prime}}_{c_{4, t, \tau}^{(i)}} \\
& +\underbrace{\widehat{\psi}_{1, \theta, 1 / 2}^{(i)}\left[\widehat{Q}_{\theta, \tau}^{(i)}\left(y_{t-1}\right)-\widehat{\psi}_{2, \theta, 1 / 2}^{(i)}\right)\left|y_{t-1}\right|}_{c_{7, t, \tau}^{(i)}}+\underbrace{\left[\widehat{\psi}_{1, \theta, \tau}^{(i)}-\widehat{\psi}_{1, \theta, 1 / 2}^{(i)}\right] \widehat{Q}_{\theta, \tau}^{(i)}\left(y_{t-1}\right)}_{c_{\theta, t, \tau}^{(i)}}
\end{aligned}
$$

The decomposition in (11) allows us to shed some light on the financial interpretation of the different $\Delta \mathrm{QL}-\mathrm{CoCaViaR}$ 's components:

- $c_{1, \tau}^{(i)}$ measures the shift in location of the system's density along the distribution of the $i$-th company, for $i=1, \ldots, N$. It quantifies the change in the system risk, when the $i$-th company moves from its median state to its $\tau$-th quantile. Such change is not attributable to the covariates of the model;

- $c_{2, t, \tau}^{(i)}$ captures the effect of the increment in the $i$-th company's risk. It corresponds to the $\Delta \mathrm{CoVaR}$, but estimated under a state of joint distress of $y_{t}$ and $x_{i, t}$;

- $c_{3, t, \tau}^{(i)}$ measures the change of the system's sensitivity to the $i$-th company. Assuming a positive conditional median for a given financial institution, positive values of $c_{3, t, \tau}^{(i)}$ suggest a larger sensitivity of the system to the $i$-th company's returns when those are in their lower quantiles;

- $c_{4, t, \tau}^{(i)}$ is the contribution associated with changes in the impact of the control variables to the system when the $i$-th company's returns move from their median to their lower quantiles;

- $c_{5, t, \tau}^{(i)}$ measures the change in the relevance of the past system's absolute returns, when the $i$-th company moves from its median to its distress state;

- $c_{6, t, \tau}^{(i)}$ allows us to assess the change in the persistence of the system's quantiles. Non-null values of $c_{6, t, \tau}^{(i)}$ suggest that the system changes its dependence on its past quantiles when contrasting the median and the distress state of the $i$-th company;

\footnotetext{
${ }^{5}$ The details about the derivation of the $\Delta \mathrm{QL}-\mathrm{CoCaViaR}$ decomposition are given in Section $\mathrm{S} 1$ of the supplementary material available online.
} 
- $c_{7, t, \tau}^{(i)}$ measures the impact of the lagged change in the risk of the system computed conditional to the $i$-th company being in a median or in a distress state. Non-null values of $c_{7, t, \tau}^{(i)}$ suggest that the system's past quantiles differ according to the state of the $i$-th company.

By jointly focusing on the different $\triangle Q L-C o C a V i a R_{t, \tau}^{(i)}$ determinants, we can isolate the impact of the elements we add to the approach proposed by Adrian and Brunnermeier (2016). In particular, if $c_{5, t, \tau}^{(i)}=c_{6, t, \tau}^{(i)}=0$, there is no impact coming from changes in the autoregressive components. Hence, the CaViaR's parameters do not depend on the state of the $i$-th company. In addition, if $\psi_{1, \theta, 1 / 2}^{(i)}=0$, the $\Delta \mathrm{QL}-\mathrm{CoCaViaR}$ loses its autoregressive behavior. If $c_{4, t, \tau}^{(i)}=0$, the role of the control variables does not depend on the state of the $i$-th company. If $c_{1, t, \tau}^{(i)}=c_{3, t, \tau}^{(i)}=0$, the impact of the $i$-th company does not depend on the location of its return within its distribution support; we then do not need to account for the quantile dependence in the evaluation of the $\triangle \mathrm{QL}-\mathrm{CoCaViaR}$. Finally, if all the determinants in (11), $c_{2, t, \tau}^{(i)}$ excluded, are equal to zero, we are back to the original proposal of Adrian and Brunnermeier (2016), adjusted for the quantile-located effects, as $\lambda_{\theta, \tau}^{(i)}$ is now estimated under a state of joint distress of $y_{t}$ and $x_{i, t}$.

\subsection{Backtesting risk measures}

We evaluate the out-of-sample performance of the risk measures described in Sections $2.1-2.2$, namely the CoVaR, the QL-CoCaViaR and the two sub-models - the CoCaViaR and the QL-CoVaR-by means of selected statistical tests and loss functions. As a standard practice, we resort to a rolling window scheme to implement the out-of-sample analysis. Given a window size of $w s$ days ending at time $t$, we first estimate the parameters of the competing models and then compute the out-of-sample risk measures. We repeat this procedure by rolling the estimation window with a step of one day ahead, until we use all of the available data. By comparing the quantile forecasts with the out-of-sample realizations of the market index, that is, $y_{t}$, for $t=w s+1, \ldots, T$, we evaluate the following hit function:

$$
H i t_{t, \theta, \tau}^{\mathcal{M} \mid i}= \begin{cases}1 & \text { if } y_{t}<\mathcal{M}_{t, \theta, \tau}^{(i)} \\ 0 & \text { otherwise }\end{cases}
$$

where $\mathcal{M}=\{\mathrm{CoVaR}, \mathrm{CoCaViaR}, \mathrm{QL}-\mathrm{CoVaR}, \mathrm{QL}-\mathrm{CoCaViaR}\}$, that is, we have four different hit functions, one for each risk model. ${ }^{6}$ Building on the hit function (12), we first compare the accuracy of the competing risk measures by implementing the Kupiec (1995) and the Christoffersen (1998) tests.

We note that the risk measures derived from a CoVaR-like framework are all conditional to the distress state of the individual companies. Nevertheless, the standard Kupiec (1995) and Christoffersen (1998) tests do not capture such a conditioning. In fact, they focus only on the quantile violation of the financial system. Therefore, we adopt the method proposed by Girardi and Ergun (2013), that

\footnotetext{
${ }^{6}$ The set of models could include a subset of our specifications or could be extended by including additional models.
} 
includes the distress state of the conditioning companies into the Kupiec (1995) and Christoffersen (1998) tests. First, we consider the hit values of the $i$-th company, defined as:

$$
H i t_{t, \tau}^{i}=\left\{\begin{array}{ll}
1 & \text { if } x_{i, t}<\widehat{Q}_{\tau}\left(x_{i, t}\right) \\
0 & \text { otherwise }
\end{array} .\right.
$$

In a second step, we compute the hit values of the system as in (12), and implement the Kupiec (1995) and Christoffersen (1998) tests for the days in which $H i t_{t, \tau}^{i}=1$ (i.e., when the $i$-th company is in distress). We refer the reader to Girardi and Ergun (2013) for further details on this test, that captures a double conditioning (i.e., the quantile violation of $y_{t}$ when $x_{i, t}$ in distress). Note that we might compute $\widehat{Q}_{\tau}\left(x_{i, t}\right)$ in (13) from different specifications, with or without the CaViaR-like dynamics (see (1) and (6)). As a result, we might obtain different distress states from (13) according to the risk measure we are using to monitor the $i$-th company's risk. Therefore, in addition to the method described above, we also implement the test proposed by Girardi and Ergun (2013) by replacing $\widehat{Q}_{\tau}\left(x_{i, t}\right)$ in (13) with $\widehat{q}_{\tau}\left(x_{i}\right)$, where $\widehat{q}_{\tau}\left(x_{i}\right)$ is the sample $\tau$-th quantile of the $i$-th company computed from the entire time series (i.e., using the observations recorded in $t=1, \ldots, T$ ). In doing so, we use a model-free hit function to identify the distress of $x_{i, t}$ and evaluate the accuracy of the four risk measures (CoVaR, CoCaViaR, QL-CoVaR and QL-CoCaViaR) on the basis of the same Hit $t_{t, \tau}^{i}$ series.

In Girardi and Ergun (2013), a financial institution can be at most at its VaR to be defined in distress, consistent with their definition of CoVaR. In our case, we are coherent with the original definition of Adrian and Brunnermeier (2016), but we are also aware that the distress state of the $i$-th company might not be confined to the case of observing its returns below a given threshold. In fact, by using the previous hit functions, we completely disregard the days in which $x_{i, t}$ is greater than $\widehat{Q}_{\tau}\left(x_{i, t}\right)$ (or $\widehat{q}_{\tau}\left(x_{i}\right)$ ), although $x_{i, t}$ could be in the right-neighborhood of its $\tau$-th quantile. We increase the flexibility in identifying the distress state of the $i$-th company, remaining, at the same time, within our modelling framework. In particular, we account for the impact exerted by $x_{i, t}$ in the neighborhood of its $\tau$-th quantile by replacing (13) with the following smooth hit function:

$$
H i t_{t, \theta}^{i}= \begin{cases}1 & \text { if } K\left(\frac{\widehat{F}_{t \mid t-1}\left(x_{i, t}\right)-\tau}{h}\right)>\nu \text { or } x_{i, t}<\widehat{Q}_{\tau}\left(x_{i, t}\right) \\ 0 & \text { otherwise }\end{cases}
$$

where $K\left(\frac{\widehat{F}_{t \mid t-1}\left(x_{i, t}\right)-\tau}{h}\right)$ is the same kernel function we use to estimate the parameters entering the quantile-located measures (see Section 2.2), whereas $\nu$ is a given threshold. As a result, we do not lose information when $x_{i, t}$ is close to its $\tau$-th quantile from the right. ${ }^{7}$

\footnotetext{
${ }^{7}$ Note that the condition $H i t_{t, \theta}^{i}=1$ if $K\left(\frac{\widehat{F}_{t \mid t-1}\left(x_{i, t}\right)-\tau}{h}\right)>\nu$ or $x_{i, t}<\widehat{Q}_{\tau}\left(x_{i, t}\right)$ is more pessimistic than $H i t_{t, \theta}^{i}=1$ if $K\left(\frac{\widehat{F}_{t \mid t-1}\left(x_{i, t}\right)-\tau}{h}\right)>\nu$. Nevertheless, we checked that the results obtained from the two hit functions, available upon
} 
Finally, we evaluate the out-of-sample performance of the four risk measures by using a loss function. A typical choice in the literature builds on the squared differences between the realizations of the system and the quantile forecasts (see, e.g., Caporin (2008)). We slightly modify this approach to take into account the distress state of the conditioning company. In particular, we first define the following weighting function:

$$
W_{t, \tau}^{i}= \begin{cases}1 & \text { if } x_{i, t} \leq \widehat{Q}_{\tau}\left(x_{i, t}\right) \\ e^{-u_{t, \tau}^{2}} & \text { otherwise }\end{cases}
$$

where $u_{t, \tau}=\left(x_{i, t}-\widehat{Q}_{\tau}\left(x_{i, t}\right)\right) / h ;(15)$ is a continuous asymmetric function at $x_{i, t}=\widehat{Q}_{\tau}\left(x_{i, t}\right)$ taking the value of 1 in the worst scenario, that is, when $x_{i, t} \leq \widehat{Q}_{\tau}\left(x_{i, t}\right)$, and smoothly decreasing for $x_{i, t}>$ $\widehat{Q}_{\tau}\left(x_{i, t}\right)$. We thus introduce the loss function:

$$
L_{t, \theta, \tau}^{\mathcal{M} \mid i}= \begin{cases}{\left[1+\left(y_{t}-\mathcal{M}_{t, \theta, \tau}^{(i)}\right)^{2}\right] W_{t, \tau}^{i}} & \text { if } y_{t}<\mathcal{M}_{t, \theta, \tau}^{(i)} \\ 0 & \text { otherwise }\end{cases}
$$

where $\mathcal{M}=\{\mathrm{CoVaR}, \mathrm{CoCaViaR}, \mathrm{QL}-\mathrm{CoVaR}, \mathrm{QL}-\mathrm{CoCaViaR}\}$. After computing (16) for the competing models, we test whether the loss differentials are statistically significant by using a Diebold and Mariano (2002)-type test.

\subsection{Backtesting $\Delta$ Risk measures}

Section 2.4 reports a set of approaches we might take into account to compare competing risk measures when evaluating, monitoring and anticipating the occurrence of extreme (tail) systemic events conditional to the distress state of individual financial institutions. However, we also use the four $\Delta$ Risk measures $\{\Delta \mathrm{CoVaR}, \Delta \mathrm{CoCaViaR}, \Delta \mathrm{QL}-\mathrm{CoVaR}$ and $\Delta \mathrm{QL}-\mathrm{CoCaViaR}\}$ to evaluate the systemic impact of individual financial institutions. As our purpose is to highlight the different informative content of these measures, we cannot simply compare the estimates of their parameters. Then, we also analyze the values they provide ex-ante combined with the ex-post market movements. In doing so, we take into account the contemporaneous movements of the conditioning financial institutions.

In this framework, the literature presents approaches for the ex-post analysis of systemic risk measures that, in general, try to identify the drivers of systemic risk. For instance, Adrian and Brunnermeier (2016) focus on the forward $-\Delta \mathrm{CoVaR}$, estimated by regressing, for each company taken individually, time-varying $\Delta$ CoVaRs on lagged institutional characteristics and common risk factors. In order to assess the out-of-sample forecasting performance of the forward- $\Delta$ CoVaR, Adrian and Brunnermeier (2016) regress, for each company taken individually, the crisis- $\Delta$ CoVaR (i.e., the $\Delta$ CoVaR computed for the period 2007:I-2008:IV) on the forward- $\Delta$ CoVaR (estimated up to 2006:IV). The request, are similar. 
out-of-sample analysis building on the forward $-\Delta \mathrm{CoVaR}$ is useful in monitoring the drivers of the systemic risk, such as lagged institutional characteristics and macroeconomic control variables. However, as stated by Adrian and Brunnermeier (2016), the forward- $\Delta$ CoVaR remains a reduced-form measure and does not allow us to causally allocate the source of systemic risk to different financial institutions. In contrast, Girardi and Ergun (2013) focus on a pre-crisis ranking of the $\Delta$ CoVaR estimated for 74 US financial institutions during the period June 2006-June 2007. Here, the $\Delta$ CoVaR values suggest that depositories are, on average, the most risky group prior to the crisis. This evidence is consistent with the findings in Billio et al. (2012), in which banks appear to be more systemically risky than other industry groups. ${ }^{8}$

Nevertheless, works comparing competing systemic risk measures, possibly nested, are not common in the literature. Indeed, to the best of our knowledge, there is no backtest in the literature specifically designed to evaluate and compare $\Delta$-type risk measures. In this section we introduce approaches to run a backtest on systemic risk measures. Following Adrian and Brunnermeier (2016), we work with $\Delta$ Risk measures in dollar terms in the backtesting exercise. This allows us to take into account the company size when evaluating the systemic risk impact. Then, we compute $\$ \Delta C o V a R_{t, \tau}^{(i)}=\Delta C o V a R_{t, \tau}^{(i)} \cdot M V_{t}^{(i)}$, where $M V_{t}^{(i)}$ is the market value of the $i$-th company at time $t$, for $i=1, \ldots, N$ and $t=1, \ldots, T$. Likewise, we obtain the series $\$ \Delta C o C a V i a R_{t, \tau}^{(i)}, \$ \Delta Q L-C o V a R_{t, \tau}^{(i)}$ and $\$ \Delta Q L-C o C a V i a R_{t, \tau}^{(i)}$. We work with two sub-samples: a training period, in which we recover ex-ante systemic risk measures, and a target period for ex-post evaluations. To compare competing $\$ \Delta$ Risk measures we start from linear correlations. First, we compute the average $\$ \Delta$ Risk measures in the training period for each company. For instance, when focusing on the CoVaR, we compute the following quantity:

$$
\overline{\$ \Delta C o V a R_{\tau}^{(i)}}=\frac{1}{M} \sum_{t=1}^{M} \$ \Delta \operatorname{CoV} a R_{t, \tau}^{(i)}
$$

where $M$ is the training sample size. Second, we move to the target period and compute the (approximate) change in the company market value as $\$ x_{i, t}=x_{i, t} \cdot M V_{t}^{(i)}$, where $x_{i, t}$ is the return of the $i$-th company. ${ }^{9}$ Then, for each company, we compute the average change in the target period, defined as follows:

$$
\overline{\$ x_{i}}=\frac{1}{K} \sum_{t=M+1}^{M+K} \$ x_{i, t},
$$

where $K$ is the size of the target sample.

The first analysis consists in computing the correlation between $\overline{\$ \Delta C o V a R_{\tau}^{(i)}}$ and $\overline{\$ x_{i}}$. We should expect large correlations, as greater absolute values of a $\$ \Delta$ Risk measure would ideally anticipate larger losses observed ex-post. Furthermore, we stress the analysis by focusing on the most risky companies.

\footnotetext{
${ }^{8}$ For completeness, we implement a similar analysis and check that banks are among the highest contributors to systemic risk, in line with Billio et al. (2012) and Girardi and Ergun (2013). Interestingly, this phenomenon becomes more evident when using the $\Delta \mathrm{QL}-\mathrm{CoCaViaR}$ (see Section S4.1 of the supplementary material available online).

${ }^{9}$ The quantity $\$ x_{i, t}$ is an approximate change in the market value of the $i$-th company as we work with log-returns.
} 
For this purpose, starting from the training period (i.e., ex-ante), we cluster the $\overline{\$ \Delta C o V a R_{\tau}^{(i)}}$ series in deciles and let the first decile (that includes the most risky companies) be the primary focus. Moving to the target period (i.e., ex-post), we cluster the $\overline{\$ x_{i}}$ series and focus again on the first decile. We then compute the linear correlation between the series in (17) and (18) by restricting the attention on the most risky companies, that is, the ones belonging to the first decile both ex-ante and ex-post. A large correlation in the first decile (that reflects the worst scenario) points out the appropriateness of a given systemic risk measure. We repeat the exercises described above for the other $\Delta$ Risk measures.

To further evaluate the out-of-sample performance of the competing $\Delta$ Risk measures, we introduce a method that builds on a sparse principal component analysis (Zou et al., 2006). We start from the deciles of the $\$ \Delta$ Risk measures computed in the training period, as described above. We then implement a two-step procedure on the target period (i.e., ex-post). In the first step, we run a principal component analysis for each $\$ \Delta$ Risk measure computed in the target period, recovering the first principal component, that is, the dominant factor. In the second step, we use the dominant factor as the response variable of a penalized regression (using the LASSO). In this model, the $N$ covariates are the original $\$ \Delta$ Risk quantities computed in the target period. We choose the optimal tuning parameter of the LASSO by using a 10-fold cross validation. We run similar regressions for each dominant factor, using as covariates the $\$ \Delta$ Risk measures corresponding to the dominant factor. Notably, such approach corresponds to a sparse principal component analysis, see Zou et al. (2006). Ex-post, after estimating the parameters, we check which of the $N$ companies significantly contribute to the systemic risk during the target period, that is, the companies that have been selected by the LASSO.

We assess the capability of a $\$ \Delta$ Risk measure to identify ex-ante the systemically important financial companies by computing a critical ratio $(C R)$. In particular, $C R$ quantifies the proportion of companies selected by the LASSO model (i.e., the companies that ex-post have a significant impact on the systemic risk) that are also classified as the most systemically important in the ex-ante ranking. As a result, the higher $C R$, the better the performance of a $\Delta$ Risk measure in identifying ex-ante the systemically important financial companies. This analysis might be performed for different lengths of the training and testing periods. Furthermore, this comparison could be easily extended to include other measures, such as the SRISK (Brownlees and Engle, 2017) and the MES (Acharya et al., 2017).

\section{Data and empirical set-up}

Our dataset includes the daily returns of 1,155 US financial institutions (952 banks and 203 insurance companies) in the period between October 10, 2000 and July 31, 2015, for a total of 3,864 days. ${ }^{10}$ Some of these companies enter the dataset after October 10, 2000, whereas others exit before July 31,

\footnotetext{
${ }^{10}$ The data are recovered from Thomson Reuters Datastream.
} 
2015. Henceforth, we consider as present the companies for which we have data on a given day between October 10, 2000 and July 31, 2015. The models described in Section 2 are estimated for each of the financial companies present in our dataset for at least 200 trading days, the number of which is equal to $1,030 .{ }^{11}$ We also build an index that reproduces the behavior of the financial system $\left(y_{t}\right)$ from the returns of the 1,155 financial institutions, weighted by their market values, from October 10, 2000 to July 31, 2015.

$\mathbf{M}_{t}$ includes control variables related to the bond, equity and real estate markets. They are listed as follows: i) the CBOE Volatility Index (VIX); ii) the liquidity spread (LS), computed as the difference between the three-month collateral repo rate and the three-month bill rate; iii) the change in the three-month Treasury bill rate (TB); iv) the change in the slope of the yield curve (YC), computed as the spread between the ten-year Treasury rate and the three-month bill rate; v) the change in the credit spread between BAA-rated bonds and the Treasury rate (CS), both with a maturity of ten years; vi) the daily equity market return (EM); vii) the excess return of the real estate sector over the market return (RE). ${ }^{12}$ The first principal component $\left(f p c_{t}\right)$ of the control variables in $\mathbf{M}_{t}$ explains $96.50 \%$ of the variability in the data. We choose to use $f p c_{t}$ in place of $\mathbf{M}_{t}$ as it allows us to exploit the almost totality of the information contained in $\mathbf{M}_{t}$, while achieving relevant benefits in terms of computational burden and estimates' stability.

We estimate the four risk measures, that is, CoVaR, CoCaViaR, QL-CoVaR and QL-CoCaViaR, using two quantile levels: $\theta=\tau=0.01$ and $\theta=\tau=0.05$. As for the estimation of both the QL-CoVaR's and the QL-CoCaViaR's parameters, we use the Gaussian kernel as $F(\cdot)$ by which we weight the observations of $x_{i, t}$ in (8). Moreover, we run a sensitivity analysis and obtain the estimates at $h=\{0.10,0.15,0.20\}$, to assess whether, and in what measure, the results change according to the choice of $h$. As for $\widehat{F}_{t \mid t-1}\left(x_{i, t}\right)$, given the values of $x_{i, t}$ and $f p c_{t-1}$ recorded in $t=2, \ldots, 51$, we estimate the quantile regression model in (1) for 50 quantile levels equally distributed $-\tau=$ $\{0.01,0.03,0.05, \ldots, 0.99\}$ - using the method proposed by Bondell et al. (2010). We then obtain 50 estimated quantiles $\widehat{Q}_{\tau}\left(x_{i, 51}\right)=\widehat{\alpha}_{\tau}^{(i)}+\widehat{\beta}_{\tau}^{(i)} f p c_{50}$ having a monotonic behavior in $\tau=\{0.01,0.03, \ldots, 0.99\}$, that we interpolate to build the conditional distribution of $x_{i, 51}$, denoted as $\widehat{F}\left(x_{i, 51} \mid f p c_{50}\right)$. Finally, we compute $\widehat{F}_{51 \mid 50}\left(x_{i, 51}\right)$ - the probability level corresponding to the realization $x_{i, 51}$ that we extrapolate from $\widehat{F}\left(x_{i, 51} \mid f p c_{50}\right)$. By using a rolling window procedure in which each estimation window includes the latest 50 values of $x_{i, t}$ and $f p c_{t-1}$, we also estimate $\widehat{F}_{t \mid t-1}\left(x_{i, t}\right)$ for $t=52, \ldots, T$.

We highlight that the conditional distribution $\widehat{F}\left(x_{i, t} \mid f p c_{t-1}\right)$ is built by interpolating 50 conditional quantiles, the parameters of which are estimated from a sample of 50 observations. In general, increasing the sample size improves the asymptotic properties of the $Q_{\tau}\left(x_{i, t}\right)$ 's estimator. Further-

\footnotetext{
${ }^{11}$ Additional details on the dataset are given in Section S2 of the supplementary material available online.

${ }^{12}$ The control variables listed in i) - v) are taken from Thomson Reuters Datastream, whereas $\mathrm{EM}$ and RE are recovered from the industry portfolios built by Kenneth R. French, available at http://mba.tuck.dartmouth.edu/pages/faculty/ken.french/data_library.html.
} 
more, using a larger set of quantile levels in $\tau \in(0,1)$ improves the accuracy of the interpolation and, as a result, leads to more accurate estimates of $\widehat{F}\left(x_{i, t} \mid f p c_{t-1}\right)$. Nevertheless, increasing both the sample size and the number of quantile levels is computationally expensive. By focusing on a subset of companies, we compare the results obtained with an estimation window of 50 observations and 50 interpolated quantiles with those obtained from a window of 200 observations and a grid of 99 quantiles- $\tau=\{0.01,0.02,0.03, \ldots, 0.99\}$. We choose the set-up with a smaller computational burden as the differences between the two approaches are negligible.

\section{Empirical findings}

\subsection{Estimation}

We estimate the risk measures' parameters on the basis of the empirical set-up described in Section $3 .^{13}$ In general, positive returns of the individual companies have a positive impact on the VaR of the financial system, as both $\lambda_{\theta}^{(i)}$ (for CoVaR and CoCaViaR) and $\lambda_{\theta, \tau}^{(i)}$ (for QL-CoVaR and QL-CoCaViaR) take, on average, positive values. Notably, the relationships between the system and the individual companies become stronger when linking the left tails of their returns' distributions, that is, when using quantile-located effects. In fact, on average, $\lambda_{\theta, \tau}^{(i)}$ is greater than $\lambda_{\theta}^{(i)}$, highlighting that the system is more sensitive to the individual companies when accentuating the distress degree in their connections. On the other hand, the CaViaR's components absorb, in part, the sensitivity of the system to the individual companies. In fact, on average, $\lambda_{\theta, \tau}^{(i)}$ and $\lambda_{\theta}^{(i)}$ decrease when including the CaViaR's components. Focusing on the CaViaR's components, the lagged quantile of the system- $Q_{\theta}^{(i)}\left(y_{t-1}\right)$ for CoCaViaR and $Q_{\theta, \tau}^{(i)}\left(y_{t-1}\right)$ for QL-CoCaViaR - has, on average, a positive impact on its current value $\left(Q_{\theta}^{(i)}\left(y_{t}\right)\right.$ or $\left.Q_{\theta, \tau}^{(i)}\left(y_{t}\right)\right)$. This reflects the persistence of quantiles over time (Engle and Manganelli, 2004). In contrast, the second CaViaR's component $\left(\left|y_{t-1}\right|\right)$ has, on average, a negative impact on the lower quantiles of the system. Therefore, a very bad or a very good day symmetrically increases the probability of observing greater losses in the next day, consistent with the hypothesis supported by Engle and Manganelli (2004). Finally, $f p c_{t}$ has, on average, a negative impact on the system.

\subsection{A comparative analysis of competing systemic risk measures}

For each day and for each company in our dataset, we compute the $\Delta$ Risk measures $-\Delta$ CoVaR, $\Delta \mathrm{CoCaViaR}, \Delta \mathrm{QL}-\mathrm{CoVaR}$ and $\Delta \mathrm{QL}-\mathrm{CoCaViaR}$. We display the trend of the cross-sectional median of each $\Delta$ Risk measure in Figure 1, distinguishing banks from insurances. ${ }^{14}$ The $\Delta$ CoCaViaR $(\Delta \mathrm{QL}-\mathrm{CoCaViaR})$ is, on average, more conservative than the $\Delta \mathrm{CoVaR}(\Delta \mathrm{QL}-\mathrm{CoVaR})$. This is due to

\footnotetext{
${ }^{13}$ We summarize here the main findings for the sake of brevity. Additional details are reported in Section S3 of the supplementary material.

${ }^{14}$ The systemic risk measures are computed at $\theta=\tau=0.01$ and $h=0.15$. The plots obtained from other values of $\theta=\tau$ and $h$, available upon request, are qualitatively similar.
} 
the CaViaR's components in both $\Delta \mathrm{CoCaViaR}$ and $\Delta \mathrm{QL}-\mathrm{CoCaViaR}$, that absorb, in part, the relations between the financial system and the individual companies (see Section 4.1). The quantile-located measures $(\Delta \mathrm{QL}-\mathrm{CoVaR}$ and $\Delta \mathrm{QL}-\mathrm{CoCaViaR})$ point out a greater extreme risk, being estimated under a state of joint distress of $y_{t}$ and $x_{i, t}$. Notably, the distance between the quantile-located measures $(\Delta \mathrm{QL}-\mathrm{CoVaR}$ and $\Delta \mathrm{QL}-\mathrm{CoCaViaR})$ and the other measures ( $\Delta$ CoVaR and $\Delta$ CoCaViaR) is relatively low during tranquil periods and becomes accentuated during stressed phases (e.g., during the subprime crisis). Smaller but still relevant spikes are observed during the 'internet bubble' in 2000, after the terrorist attacks in September 2001, around the stock market crash in 2002, during the war in Iraq (2001 - 2003) and the European sovereign debt crisis (2010 - 2011). We then highlight the capability of the quantile-located measures to react more strongly during events of system-wide relevance.
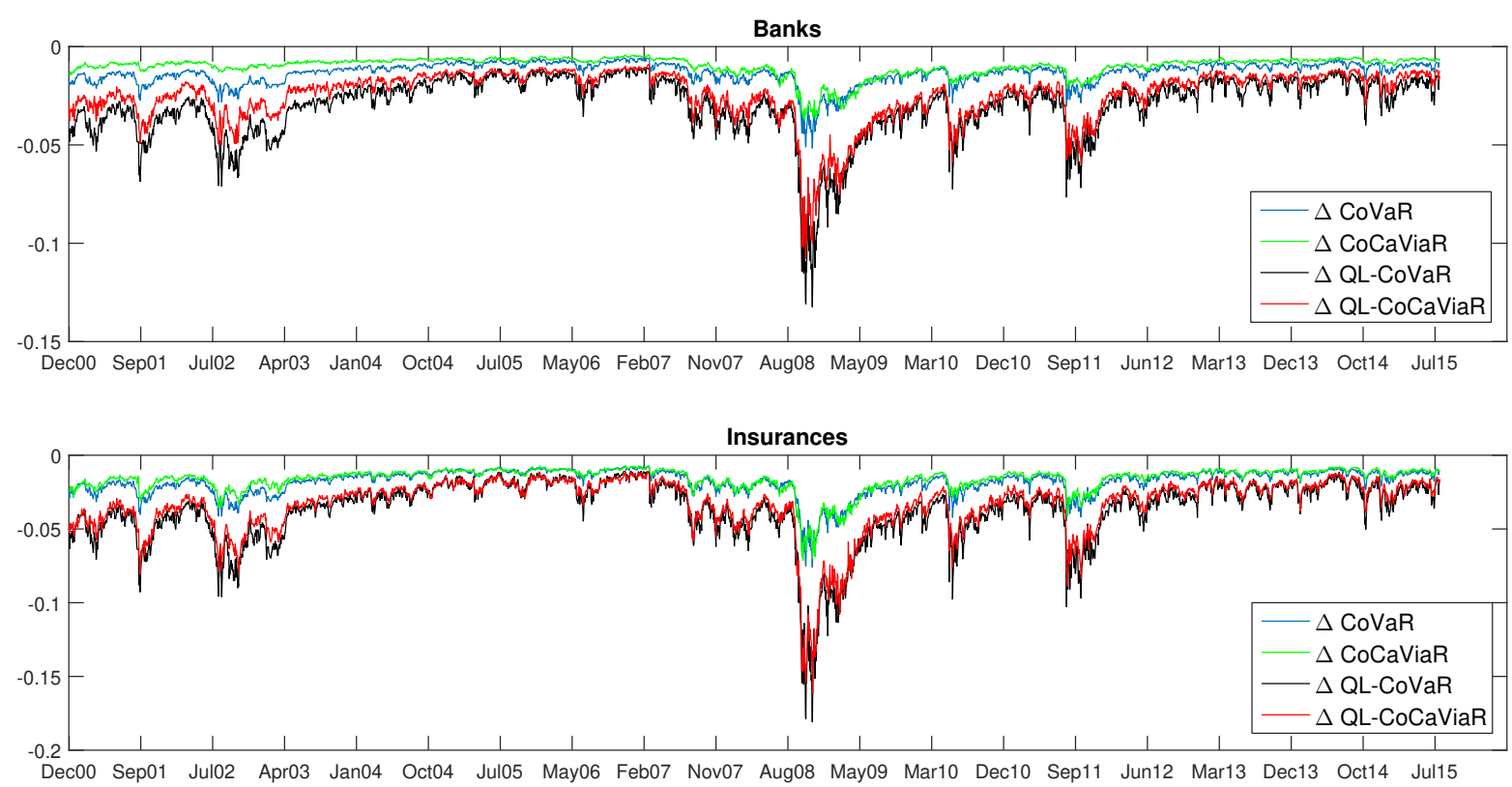

Figure 1: For each day in the period December 2000-July 2015, the figure displays the trend of the cross-sectional medians of the following risk measures: $\Delta \mathrm{CoVaR}, \Delta \mathrm{CoCaViaR}, \Delta \mathrm{QL}-\mathrm{CoVaR}$ and $\Delta \mathrm{QL}-\mathrm{CoCaViaR}$, distinguishing banks from insurances. The risk measures are computed at $\theta=\tau=0.01$ and $h=0.15$. The results obtained at $\theta=\tau=0.05$ and $h=\{0.10,0.20\}$, available upon request, are qualitative similar.

From a systemic risk perspective, the absolute values of the quantile-located risk measures are greater than the standard CoVaR. Nevertheless, these differences do not correspond to simple shifts but tend to increase during periods of market distress. ${ }^{15}$ Therefore, the introduction of quantile-located effects does not simply provide a shift in the estimated systemic impact; it also accounts for the change in the structural relations between the individual companies and the market. This is evident when the overall system is in turmoil. As a result, risk measures building on quantile-location are more reactive to the occurrence of extreme events and, thus, more appealing for regulators and risk managers.

For each company, we compute the 5-th and the 95-th percentiles, the median and the interquartile

\footnotetext{
${ }^{15}$ See also Figure S3 in the supplementary material.
} 
range of each risk measure's time series. We then calculate the cross-sectional medians of these statistics, as reported in Table 1. The CaViaR's components bring some differences between the $\triangle \mathrm{CoVaR}$ and the $\Delta \mathrm{CoCaViaR}$. Nevertheless, the sign of these differences is not constant and depends on the $\theta$ levels. For instance, at $\theta=\tau=0.01, \Delta C o V a R_{t, \tau}^{(i)}$ is greater, in absolute value, than $\Delta C o C a V i a R_{t, \tau}^{(i)}$ in its 5 -th and 95-th percentiles and at the median level. Hence, at $\theta=\tau=0.01$, $\triangle C o V a R_{t, \tau}^{(i)}$ points out a greater marginal contribution of the companies to the systemic risk with respect to $\Delta C o C a V i a R_{t, \tau}^{(i)}$. The opposite phenomenon holds at $\theta=\tau=0.05: \Delta C o C a V i a R_{t, \tau}^{(i)}$ reflects a greater risk with respect to $\Delta C o V a R_{t, \tau}^{(i)}$. Furthermore, $\Delta C o C a V i a R_{t, \tau}^{(i)}$ is slightly more volatile than $\Delta C o V a R_{t, \tau}^{(i)}$, as we can see from the interquartile range.

Table 1: Statistics of $\Delta$ CoVaR, $\Delta$ CoCaViaR, $\Delta$ QL-COVaR and $\Delta$ QL-CoCaViaR

\begin{tabular}{c|cccc|cccc}
\hline & \multicolumn{4}{|c}{$\theta=\tau=0.01$} & \multicolumn{4}{c}{$\theta=\tau=0.05$} \\
\hline & $5 \mathrm{P}$ & MED & $95 \mathrm{P}$ & IQR & $5 \mathrm{P}$ & MED & $95 \mathrm{P}$ & IQR \\
\hline$\Delta$ CoVaR,$\tau$ & -1.022 & -0.645 & -0.468 & 0.215 & -0.496 & -0.306 & -0.228 & 0.095 \\
$\Delta$ CoCaViaR & -0.990 & -0.488 & -0.263 & 0.255 & -0.839 & -0.417 & -0.229 & 0.218 \\
$\Delta$ QL-CoVaR & -2.815 & -1.442 & -0.741 & 0.970 & -1.605 & -0.704 & -0.348 & 0.538 \\
$\Delta$ QL-CoCaViaR & -3.112 & -1.088 & 0.003 & 1.251 & -2.015 & -0.613 & -0.019 & 0.712 \\
\hline
\end{tabular}

For each day and for each company in our dataset, we compute the four risk measures given in the first column, setting $\theta=\tau=\{0.01,0.05\}$ and $h=0.15$ (the results obtained for other bandwidth values, available upon request, are similar). For each company, we then compute the 5 -th percentile (5P, \%), the median (MED, \%), the 95-th percentile (95P, \%) and the interquartile range (IQR, \%) of the four risk measures' time series. Finally, we report from left to right the cross-sectional medians of these statistics.

All the statistics in Table 1 sensibly increase, in absolute value, when considering $\Delta \mathrm{QL}-\mathrm{CoVaR}$ and $\Delta \mathrm{QL}-\mathrm{CoCaViaR}$, which reflect a larger systemic risk contribution coming from the individual companies. This is due to the fact that $\triangle Q L-C o V a R_{t, \tau}^{(i)}$ and $\triangle Q L-C o C a V i a R_{t, \tau}^{(i)}$ are estimated under a state of joint distress of $y_{t}$ and $x_{i, t}$. Besides, on average, $\triangle Q L-C o V a R_{t, \tau}^{(i)}$ is greater (in absolute value) than $\triangle Q L-C o C a V i a R_{t, \tau}^{(i)}$. Indeed, the CaViaR's components in $\triangle Q L-C o C a V i a R_{t, \tau}^{(i)}$ absorb, in part, the relationships between $y_{t}$ and $x_{i, t}$ in their quantiles, lowering the contribution of the individual companies to the systemic risk. ${ }^{16}$ The interquartile range increases when including quantile-located effects. This is due to the fact that the estimates of both $\triangle Q L-C o V a R_{t, \tau}^{(i)}$ and $\triangle Q L-C o C a V i a R_{t, \tau}^{(i)}$ are located, day by day, within the neighborhood of the $\tau$-th $x_{i, t}$ 's quantile. As discussed in Section 2.2, the quantiles (and thus the distribution) of $x_{i, t}$ change over time and these variations contribute to the volatility of both $\triangle Q L-C o V a R_{t, \tau}^{(i)}$ and $\triangle Q L-C o C a V i a R_{t, \tau}^{(i)}$.

Furthermore, we also compare the four $\Delta$ Risk measures by using the following indicator:

$$
Z_{j, k, \tau}=\frac{1}{T N} \sum_{i=1}^{N} \sum_{t=1}^{T} \mathbf{1}_{\left\{\Delta \operatorname{Risk}_{j, t, \tau}^{(i)}-\Delta R i s k_{k, t, \tau}^{(i)}<-\phi\right\}}
$$

where $\Delta R i s k_{j, t, \tau}^{(i)}$ and $\Delta R i s k_{k, t, \tau}^{(i)}$ are the $j$-th and the $k$-th measures in the set $S_{t, \tau}^{(i)}=\left\{100 \cdot \Delta C o V a R_{t, \tau}^{(i)}\right.$,

\footnotetext{
${ }^{16}$ This is consistent with the analysis of the risk measures' coefficients in Sections 4.1 and S3 (supplementary material).
} 
$\left.100 \cdot \Delta \operatorname{CoCaViaR}(i), 100 \cdot \Delta Q L-C o V a R_{t, \tau}^{(i)}, 100 \cdot \Delta Q L-C o C a V i a R_{t, \tau}^{(i)}\right\}$, for $j=1, \ldots, 4$ and $k=1, \ldots, 4$, $\mathbf{1}_{\{\cdot\}}$ is an indicator function taking the value of 1 if the condition in $\{\cdot\}$ is true, the value of 0 otherwise, whereas $\phi$ is a given threshold. $Z_{j, k, \tau}=0$ if $k=j$.

$Z_{j, k, \tau}$ in (19) quantifies the proportion of times - over $T$ and $N$-in which the $j$-th systemic risk measure signals a worsening in the riskiness with respect to the $k$-th indicator. We neglect minimal differences, therefore $Z_{j, k, \tau}$ captures a given worsening if, and only if, its magnitude is greater, in absolute value, than $\phi$. Here, we set $\phi$ equal to 10 basis points and report the results - obtained at $\theta=\tau=\{0.01,0.05\}$ - in Table 2. In each of the two panels of Table $2, Z_{j, k, \tau}$ is computed as the difference between the risk measure in the $j$-th row and the risk measure in the $k$-th column, for $j, k=1, \ldots, 4$.

Table 2: Indicator $Z_{j, k, \tau}$

\begin{tabular}{|c|c|c|c|c|}
\hline & \multicolumn{4}{|c|}{$\tau=0.01$} \\
\hline & $\Delta \mathrm{CoVaR} R_{\tau}$ & $\Delta C o C a V i a R_{\tau}$ & $\Delta Q L-C o V a R_{\tau}$ & $\Delta Q L-C o C a V i a R_{\tau}$ \\
\hline \multirow{6}{*}{$\begin{array}{l}\Delta \mathrm{CoVaR}_{\tau} \\
\Delta \mathrm{CoCaViaR_{ \tau }} \\
\Delta Q L-\mathrm{CoVaR} \\
\Delta Q L-C o C a V i a R_{\tau}\end{array}$} & 0.000 & 45.726 & 21.335 & 30.195 \\
\hline & 23.614 & 0.000 & 21.363 & 26.436 \\
\hline & 71.861 & 72.503 & 0.000 & 54.264 \\
\hline & 62.479 & 67.098 & 35.676 & 0.000 \\
\hline & \multicolumn{4}{|c|}{$\tau=0.05$} \\
\hline & $\Delta \mathrm{CoVaR} R_{\tau}$ & $\Delta C o C a V i a R_{\tau}$ & $\Delta Q L-C o V a R_{\tau}$ & $\Delta Q L-C o C a V i a R_{\tau}$ \\
\hline$\Delta C o V a R_{\tau}$ & 0.000 & 9.062 & 9.334 & 19.814 \\
\hline$\Delta \mathrm{CoCaViaR_{ \tau }}$ & 58.445 & 0.000 & 31.706 & 40.021 \\
\hline$\Delta Q L-C o V a R_{\tau}$ & 73.947 & 47.930 & 0.000 & 44.645 \\
\hline$\Delta Q L-C o C a V i a R_{\tau}$ & 63.608 & 41.565 & 32.864 & 0.000 \\
\hline
\end{tabular}

The table displays the values of $Z_{j, k, \tau}$ defined in (19) for each of the pairs of the risk measures ordered, respectively, in the $j$-th row and in the $k$-th column, for $j, k=1, \ldots, 4$.

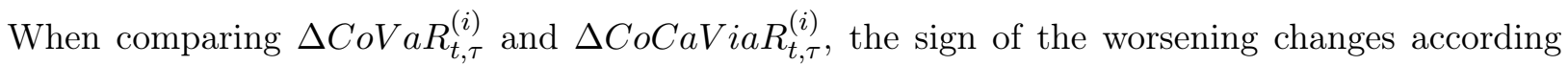
to the value of $\tau$. In fact, on average, $\Delta C o V a R_{t, \tau}^{(i)}$ provides a greater proportion of worsening with respect to $\Delta C o C a V i a R_{t, \tau}^{(i)}$ at $\tau=0.01$ (45.73\% versus $23.61 \%$; see Table 2 ). The opposite holds at $\tau=$ $0.05-\Delta C o C a V i a R_{t, \tau}^{(i)}$ signals a larger worsening than $\Delta C o V a R_{t, \tau}^{(i)}(58.45 \%$ versus $9.06 \%)$. However, both $\triangle C o V a R_{t, \tau}^{(i)}$ and $\Delta C o C a V i a R_{t, \tau}^{(i)}$ provide lower proportions of worsening in the systemic risk when compared with the quantile-located measures. In fact, the highest values of $Z_{j, k, \tau}$ in Table 2 are observed at $j=\{3,4\}$-i.e., when considering either $\Delta \mathrm{QL}-\mathrm{CoVaR}$ or $\Delta \mathrm{QL}-\mathrm{CoCaViaR}$ as $\Delta \operatorname{Risk}_{j, t, \tau}^{(i)}$ in (19). This evidence is consistent with the results discussed above: the relationships between $y_{t}$ and $x_{i, t}$ in their lower quantiles become more accentuated when including quantile-located effects; besides, the CaViaR's components absorb, in part, the impact of the individual companies on the system.

Figure 2 provides an example for four selected companies: JP Morgan Chase \& Co., Bank of America, Aflac and American International Group. Here, the quantile-located measures $(\Delta \mathrm{QL}-\mathrm{CoVaR}$ and $\Delta \mathrm{QL}-\mathrm{CoCaViaR}$ ) almost always point out a greater risk with respect to $\Delta$ CoVaR. Nevertheless, the difference between the quantile-located measures and the $\Delta \mathrm{CoVaR}$ is not constant over time. As 

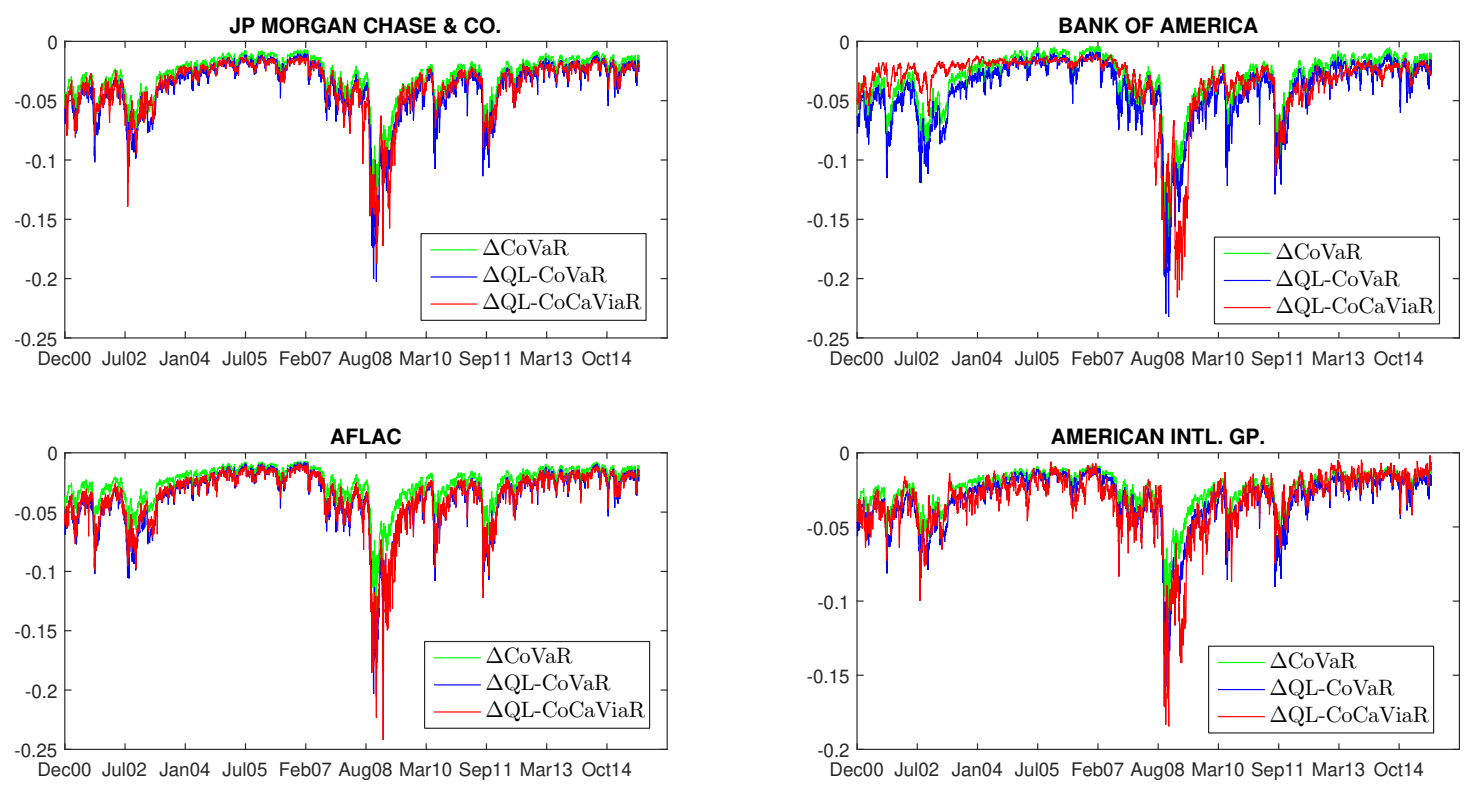

Figure 2: The four panels display the trend of $\Delta \mathrm{CoVaR}, \Delta \mathrm{QL}-\mathrm{CoVaR}$ and $\Delta \mathrm{QL}-\mathrm{CoCaViaR}$ for two selected banks and two selected insurance companies. The risk measures are computed at $\theta=\tau=0.01$ and $h=0.15$.

described above, the quantile-located measures do not simply provide a linear downwards shift of risk with respect to the $\Delta \mathrm{CoVaR}$, but account for changes in the structural relations, especially during crisis periods. Besides, we can also find periods in which the $\Delta \mathrm{CoVaR}$ is greater (in absolute value) than the quantile-located measures. This opens the door for further analyses about the opportunity cost associated with the possible precautionary immobilization of financial resources to offset the impact of systemic events.

The interconnections among financial institutions become critical during special events, such as financial crises. Therefore, it is interesting to assess how the statistics of the $\Delta$ Risk measures react to the occurrence of such events and, for this purpose, we focus on the subprime crisis. Hence, we compute the four $\Delta$ Risk measures from the data recorded in September 2008, reporting the descriptive statistics in Table 3. On average, the 5-th and the 95-th percentiles as well as the median of the four measures significantly change with respect to the full sample results, highlighting a greater contribution of the financial companies to the systemic risk during the subprime crisis. $\triangle Q L-C o V a R_{t, \tau}^{(i)}$ and $\Delta Q L-C o C a V i a R_{t, \tau}^{(i)}$ are, on average, the most sensitive to the subprime crisis, recording the highest percentage variations in their medians with respect to the full sample results. We then have a further evidence that the quantile-located relationships are critical during crisis periods, being important signaling tools to prevent or mitigate the effects of extreme events. ${ }^{17}$

We now decompose $\Delta Q L-C o C a V i a R_{t, \tau}^{(i)}$ defined in (11) into the components $c_{j, t, \tau}^{(i)}$, for $j=1, \ldots, 7$, as discussed in Section 2.3. We evaluate the relevance of the various elements we add to the stan-

\footnotetext{
${ }^{17}$ See Section S4.2 of the supplementary material for additional evidences about the quantile-location relevance in systemic risk analyses.
} 
Table 3: $\Delta$ Risk measures during the subprime crisis

\begin{tabular}{c|cccc|cccc}
\hline & \multicolumn{4}{|c}{$\theta=\tau=0.01$} & \multicolumn{4}{c}{$\theta=\tau=0.05$} \\
\hline & $5 \mathrm{P}$ & MED & 95P & IQR & 5P & MED & 95P & IQR \\
\hline$\Delta$ CoVaR $R_{t, \tau}$ & -2.232 & -1.669 & -1.209 & 0.524 & -1.355 & -1.004 & -0.720 & 0.303 \\
$\Delta$ CoCaViaR & -1.776 & -1.215 & -0.781 & 0.490 & -2.105 & -1.445 & -1.026 & 0.575 \\
$\Delta Q L-$ CoVaR & -6.870 & -4.890 & -3.094 & 1.863 & -3.820 & -2.592 & -1.623 & 1.120 \\
$\Delta Q L-$ CoCaViaR & -6.440 & -3.443 & -1.540 & 2.565 & -4.540 & -2.879 & -1.468 & 1.787 \\
\hline
\end{tabular}

For the days in September 2008 and for each company in our dataset, we compute the four risk measures given in the first column setting $\theta=\tau=\{0.01,0.05\}$ and $h=0.15$ (the results obtained for other bandwidth values, available upon request, are similar). For each company, we then compute the 5-th percentile (5P, \%), the median (MED, \%), the 95-th percentile $(95 \mathrm{P}, \%)$ and the interquartile range (IQR, \%) of the four risk measures. Finally, we report, from left to right, the cross-sectional medians of the four descriptive statistics.

dard $\triangle$ CoVaR by measuring their relative contribution to $\Delta Q L-C o C a V i a R_{t, \tau}^{(i)}$. In particular, we compute the weights of the seven components as: $w_{1, t, \tau}^{(i)}=c_{1, \tau}^{(i)} /\left|\Delta Q L-C o C a V i a R_{t, \tau}^{(i)}\right|$ and $w_{j, t, \tau}^{(i)}=$ $c_{j, t, \tau}^{(i)} /\left|\Delta Q L-C o C a V i a R_{t, \tau}^{(i)}\right|$, for $j=2, \ldots, 7$. In doing so, we can compare, on the one hand, the magnitude of the weights of the seven components. On the other hand, we can asses the sign of their contribution - to understand whether each component moves the $\triangle \mathrm{QL}-\mathrm{CoCaViaR}$ leftwards or rightwards. For each company and for each time series of the weights, we compute the 5 -th and the 95-th percentiles, the median and the interquartile range. Then, we report the cross-sectional medians of these statistics in Table $4 .^{18}$

Table 4 shows that the second component of the $\Delta \mathrm{QL}-\mathrm{CoCaViaR}$ (the traditional $\Delta$ CoVaR revised under a quantile-located perspective) provides the most relevant contribution. Notably, $c_{2, t, \tau}^{(i)}$ is the core of $\triangle Q L-C o C a V i a R_{t, \tau}^{(i)}$, as it measures the impact of the increment in the risk of the $i$-th company. We stress that the contribution of this component would have been less relevant without considering the quantile-located relations. In fact, the cross-sectional median of $\hat{\lambda}_{\theta, \tau}^{(i)}$ - entering both QL-CoVaR and QL-CoCaViaR - is greater, on average, than $\hat{\lambda}_{\theta}^{(i)}$ — entering both CoVaR and CoCaViaR, that is, the risk measures which do not include quantile-located effects. ${ }^{19}$ As a result, on average, the following inequality holds: $\left|\widehat{\lambda}_{\theta, \tau}^{(i)}\left[\widehat{Q}_{\tau}\left(x_{i, t}\right)-\widehat{Q}_{1 / 2}\left(x_{i, t}\right)\right]\right|>\left|\widehat{\lambda}_{\theta}^{(i)}\left[\widehat{Q}_{\tau}\left(x_{i, t}\right)-\widehat{Q}_{1 / 2}\left(x_{i, t}\right)\right]\right|$. It is interesting to observe that $w_{2, t, \tau}^{(i)}$ - the weight of $c_{2, t, \tau}^{(i)}$-increases, on average, when moving from $\theta=\tau=0.05$ to $\theta=\tau=0.01$, that is, when increasing the distress degree in the financial connections.

The impact of the individual companies on the $\Delta \mathrm{QL}-\mathrm{CoCaViaR}$ depends also on $c_{3, t, \tau}^{(i)}$, that is, the median quantile of $x_{i, t}-Q_{1 / 2}\left(x_{i, t}\right)$-times the residual impact of the $i$-th company when moving from the left tail to the center of the $x_{i, t}$ 's distribution- $\left(\lambda_{\theta, \tau}^{(i)}-\lambda_{\theta, 1 / 2}^{(i)}\right)$. Nevertheless, the weight of $c_{3, t, \tau}^{(i)}$ is close to zero (see Table 4). This is due to the fact that the median of the $i$-th company's returns - $Q_{1 / 2}\left(x_{i, t}\right)$-is close to zero. As a result, the impact of the individual companies to the systemic risk is entirely captured by $c_{2, t, \tau}^{(i)}$.

\footnotetext{
${ }^{18}$ We also display the time evolution of the cross-sectional medians of the seven ratios in Figures S4-S5 given in the supplementary material.

${ }^{19}$ See Section 4.1 and Section S3 of the supplementary material available online.
} 
We check that the persistence of the $y_{t}$ 's quantiles is affected by the co-movements between the system and the companies. Furthermore, the relevance of these co-movements changes according to the state in which the system and the individual companies are located. Indeed, on average, $\psi_{1,0.01,0.01}^{(i)}$ is less than half $\psi_{1,0.01,1 / 2}^{(i)}$, whereas the difference between $\psi_{1,0.05,0.05}^{(i)}$ and $\psi_{1,0.05,1 / 2}^{(i)}$ is almost imperceptible (see Table S6 given in the supplementary material). This result is reflected in $c_{6, t, \tau}^{(i)}=$ $\left[\psi_{1, \theta, \tau}^{(i)}-\psi_{1, \theta, 1 / 2}^{(i)}\right] Q_{\theta, \tau}^{(i)}\left(y_{t-1}\right)$, whose contribution to $\Delta Q L-C o C a V i a R_{t, \tau}^{(i)}$ is greater at $\theta=\tau=0.01$ - the worst scenario - than at $\theta=\tau=0.05$. Despite the relevant average value of $\psi_{1, \theta, 1 / 2}^{(i)}$, mainly at

Table 4: Statistics of the weights of the $\Delta \mathrm{QL}-\mathrm{CoCaViaR}$ 's components

\begin{tabular}{|c|c|c|c|c|c|c|c|c|}
\hline WEIGHTS & $5 \mathrm{P}$ & MED & $95 \mathrm{P}$ & IQR & $5 \mathrm{P}$ & MED & $95 \mathrm{P}$ & IQR \\
\hline & \multicolumn{4}{|c|}{$\theta=\tau=0.01, h=0.10$} & \multicolumn{4}{|c|}{$\theta=\tau=0.05, h=0.10$} \\
\hline$w_{1, \tau}$ & -101.856 & -35.819 & -11.609 & 95.065 & -48.587 & -16.754 & -5.388 & 89.402 \\
\hline$w_{2, \tau}$ & -131.081 & -57.298 & -26.414 & 41.808 & -144.169 & -54.247 & -20.422 & 35.963 \\
\hline$w_{3, \tau}$ & 0.000 & 0.000 & 0.000 & 0.000 & 0.000 & 0.000 & 0.000 & 0.000 \\
\hline$w_{4, \tau}$ & -82.256 & 2.045 & 98.687 & 73.300 & -72.392 & 3.351 & 95.588 & 73.746 \\
\hline$w_{5, \tau}$ & 0.084 & 4.283 & 21.818 & 23.833 & 0.032 & 1.513 & 7.336 & 20.949 \\
\hline$w_{6, \tau}$ & 1.116 & 22.366 & 56.510 & 75.889 & -5.157 & 6.671 & 19.418 & 65.378 \\
\hline \multirow[t]{2}{*}{$w_{7, \tau}$} & -61.971 & -2.708 & 64.181 & 44.555 & -70.861 & -1.603 & 76.039 & 40.945 \\
\hline & \multicolumn{4}{|c|}{$\theta=\tau=0.01, h=0.15$} & \multicolumn{4}{|c|}{$\theta=\tau=0.05, h=0.15$} \\
\hline$w_{1, \tau}$ & -82.921 & -29.271 & -10.113 & 78.493 & -25.660 & -10.464 & -3.313 & 72.051 \\
\hline$w_{2, \tau}$ & -134.561 & -67.777 & -33.145 & 40.591 & -143.669 & -62.208 & -24.930 & 34.405 \\
\hline$w_{3, \tau}$ & 0.000 & 0.000 & 0.000 & 0.000 & 0.000 & 0.000 & 0.000 & 0.000 \\
\hline$w_{4, \tau}$ & -72.173 & 2.344 & 86.350 & 67.441 & -54.310 & 2.847 & 74.098 & 56.549 \\
\hline$w_{5, \tau}$ & 0.076 & 3.487 & 16.212 & 19.922 & 0.035 & 1.443 & 6.146 & 18.625 \\
\hline$w_{6, \tau}$ & 1.970 & 22.110 & 54.088 & 64.325 & -4.347 & 3.583 & 11.440 & 53.354 \\
\hline \multirow[t]{2}{*}{$w_{7, \tau}$} & -53.781 & -1.309 & 63.991 & 42.919 & -61.939 & -1.295 & 60.295 & 33.211 \\
\hline & \multicolumn{4}{|c|}{$\theta=\tau=0.01, h=0.20$} & \multicolumn{4}{|c|}{$\theta=\tau=0.05, h=0.20$} \\
\hline$w_{1, \tau}$ & -33.028 & -15.017 & -5.663 & 61.374 & -14.577 & -6.544 & -2.239 & 48.040 \\
\hline$w_{2, \tau}$ & -134.641 & -81.957 & -41.311 & 38.337 & -146.176 & -75.290 & -33.567 & 32.389 \\
\hline$w_{3, \tau}$ & 0.000 & 0.000 & 0.000 & 0.000 & 0.000 & 0.000 & 0.000 & 0.000 \\
\hline$w_{4, \tau}$ & -56.540 & 1.211 & 70.667 & 55.939 & -39.682 & 2.687 & 61.254 & 44.534 \\
\hline$w_{5, \tau}$ & 0.064 & 3.125 & 14.809 & 15.729 & 0.032 & 1.473 & 5.815 & 16.004 \\
\hline$w_{6, \tau}$ & 0.630 & 13.963 & 30.293 & 54.516 & -2.641 & 3.148 & 8.471 & 39.197 \\
\hline$w_{7, \tau}$ & -48.770 & -0.677 & 54.998 & 38.114 & -52.468 & -0.598 & 42.306 & 25.688 \\
\hline
\end{tabular}

For each day and for each company, we compute the weights of the $\Delta \mathrm{QL}-\mathrm{CoCaViaR}$ 's components. For each company and for each time series of the weights, we then compute the following statistics: the 5-th (5P, \%) and the 95-th (95P, \%) percentiles, the median (MED, \%) and the interquartile range (IQR, \%). The table reports the cross-sectional medians of these statistics.

$\theta=\tau=0.01$ (see Table S6), the weight of $c_{7, t, \tau}^{(i)}=\psi_{1, \theta, 1 / 2}^{(i)}\left[Q_{\theta, \tau}^{(i)}\left(y_{t-1}\right)-Q_{\theta, 1 / 2}^{(i)}\left(y_{t-1}\right)\right]$ is relatively low. $c_{1, \tau}^{(i)}$ is always negative and more relevant at $\theta=\tau=0.01$. In contrast, $c_{4, t, \tau}^{(i)}$ and $c_{5, t, \tau}^{(i)}$ take, on average, positive values and do not significantly change between $\theta=\tau=0.01$ and $\theta=\tau=$ 0.05. We remind the reader that the last three components measure the changes in location of the system's density $\left(c_{1, \tau}^{(i)}\right)$, the changes in the impact of the control variables $\left(c_{4, t, \tau}^{(i)}\right)$ and the changes in the relevance of the past returns of the system $\left(c_{5, t, \tau}^{(i)}\right)$, when moving along the marginal distributions of the conditioning companies. Interestingly, the sign of the $c_{4, t, \tau}^{(i)}$ 's contribution is not constant over time (see Figures S4-S5). In particular, $c_{4, t, \tau}^{(i)}$ increases the systemic risk during market turmoils (e.g., the end of the technology market bubble, the 11th of September 2001, during the subprime crisis and 
during the European sovereign debt crisis). In contrast, we observe the opposite effect when markets are upward trending (e.g., the period 2003-2006). This evidence suggests that the QL-CoCaViaR provides relevant insights on the role of control variables during turmoils. This is consistent with the interpretation of these events as systemic, given that the deterioration of the overall market conditions might further contribute to the systemic risk through a set of control or state variables. Summing up, the findings discussed above highlight the importance of the additional elements we add to the standard $\Delta \mathrm{CoVaR}$. The new components take into account the persistence of the conditional quantiles over time (CoCaViaR), the quantile-located effects (QL-CoVaR) and their combination (QL-CoCaViaR).

\subsection{Backtesting results}

Table 5: Unconditional and conditional coverage tests

\begin{tabular}{c|cccc|cccc}
\hline & \multicolumn{3}{|c}{ UNCONDITIONAL COVERAGE TEST } & \multicolumn{4}{c}{ CONDITIONAL COVERAGE TEST } \\
\hline NOBS & CoVaR & CoCaViaR & QL-CoVaR & QL-CoCaViaR & CoVaR & CoCaViaR & QL-CoVaR & QL-CoCaViaR \\
\hline 100 & 38.422 & 43.511 & 32.353 & 39.386 & 38.931 & 43.003 & 31.714 & 34.015 \\
200 & 36.063 & 41.876 & 30.000 & 37.467 & 36.988 & 41.347 & 29.200 & 31.600 \\
300 & 32.587 & 38.741 & 26.346 & 34.703 & 33.427 & 38.322 & 25.354 & 28.329 \\
400 & 30.891 & 37.213 & 25.109 & 33.527 & 31.753 & 36.782 & 24.093 & 27.141 \\
\hline
\end{tabular}

For each of the four risk measures, that is, CoVaR, CoCaViaR, QL-CoVaR and QL-CoCaViaR, the table reports the percentages of times over the cross-section and over time in which the null hypothesis of the unconditional coverage test by Kupiec (1995) and the null hypothesis of the conditional coverage test by Christoffersen (1998) are not rejected at the $1 \%$ significance level, when we have at least NOBS number of observations for each series in (12). CoVaR, CoCaViaR, $\mathrm{QL}-\mathrm{CoVaR}$ and QL-CoCaViaR are computed by setting $\theta=\tau=0.05$ and $h=0.15$.

The out-of-sample accuracy of $C o V a R_{t, \theta, \tau}^{(i)}, C o C a V i a R_{t, \theta, \tau}^{(i)}, Q L-C o V a R_{t, \theta, \tau}^{(i)}$ and $Q L-C o C a V i a R_{t, \theta, \tau}^{(i)}$ is evaluated using the backtesting methods described in Section 2.4. In particular, we implement a rolling window procedure by setting $w s=300$. As for the estimates, we set $\theta=\tau=0.05$ and $h=0.15 .^{20}$ We first compare the predictive accuracy of the four risk measures using the unconditional and the conditional coverage tests, without conditioning the hit of the system to the distress state of the individual financial companies. Table 5 reports the percentages of times (over time and over the cross-section) in which we do not reject the null hypothesis of the tests at the $1 \%$ significance level. Given the features of our dataset, we implement the tests on the series for which we have at least 100, 200, 300 and 400 observations for the hit functions. We note that $C o C a V i a R_{t, 0.05,0.05}^{(i)}$ records the highest percentages of non-rejections.

We now repeat the same exercise by implementing the Kupiec (1995) and the Christoffersen (1998) tests during the subprime crisis. Table 6 reports the results obtained in four different time intervals, differing in their endpoint: 01/07/2008 - 30/09/2008, 01/07/2008 - 31/10/2008, 01/07/2008—30/11/2008 and $01 / 07 / 2008-31 / 12 / 2008$. $Q L-C o C a V_{i a R}(i), 0.05,0.05$ records the best performance when we use the shortest interval $(01 / 07 / 2008$ - 30/09/2008) for both the unconditional and the conditional coverage

\footnotetext{
${ }^{20}$ The results obtained with other values of $\theta, \tau$ and $h$ are available upon request.
} 
Table 6: Unconditional and conditional coverage tests during the subprime crisis

\begin{tabular}{c|cccc|cccc}
\hline & \multicolumn{3}{|c|}{ UNCONDITIONAL COVERAGE TEST } & \multicolumn{3}{c}{ CONDITIONAL COVERAGE TEST } \\
\hline PERIOD & CoVaR & CoCaViaR & QL-CoVaR & QL-CoCaViaR & CoVaR & CoCaViaR & QL-CoVaR & QL-CoCaViaR \\
\hline Jul 08-Sep 08 & 47.395 & 65.509 & 60.150 & 69.424 & 47.643 & 66.501 & 60.652 & 72.431 \\
Jul 08-Oct 08 & 62.879 & 81.061 & 75.255 & 76.786 & 62.879 & 82.323 & 76.276 & 80.612 \\
Jul 08-Nov 08 & 70.229 & 75.064 & 84.062 & 73.779 & 70.738 & 78.626 & 85.090 & 75.578 \\
Jul 08-Dec 08 & 84.103 & 84.872 & 89.637 & 78.497 & 84.872 & 90.769 & 91.710 & 83.161 \\
\hline
\end{tabular}

For each of the four risk measures, that is, CoVaR, CoCaViaR, QL-CoVaR and QL-CoCaViaR, the table reports the percentages of times over the cross-section and over time in which the null hypothesis of the unconditional coverage test by Kupiec (1995) and the null hypothesis of the conditional coverage test by Christoffersen (1998) are not rejected at the $1 \%$ significance level. The tests are implemented using four different periods: 01/07/2008 - 30/09/2008, 01/07/2008 - 31/10/2008, 01/07/2008 - 30/11/2008 and 01/07/2008 - 31/12/2008. CoVaR, CoCaViaR, QL-CoVaR and QL-CoCaViaR are computed by setting $\theta=\tau=0.05$ and $h=0.15$.

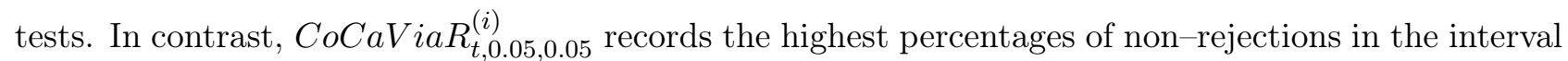
$01 / 07 / 2008$ - 31/10/2008, whereas $Q L-C o V a R_{t, 0.05,0.05}^{(i)}$ overperforms the other competing measures in the remaining periods.

Table 7: Coverage tests at the distress state of the conditioning companies

\begin{tabular}{c|cccc|cccc}
\hline & \multicolumn{3}{|c}{ UNCONDITIONAL COVERAGE TEST } & \multicolumn{4}{c}{ CONDITIONAL COVERAGE TEST } \\
\hline PERIOD & CoVaR & CoCaViaR & QL-CoVaR & QL-CoCaViaR & CoVaR & CoCaViaR & QL-CoVaR & QL-CoCaViaR \\
\hline GE-1 & 28.172 & 30.125 & 27.736 & 29.910 & 28.731 & 28.877 & 34.528 & 25.946 \\
GE-2 & 54.225 & 47.042 & 66.857 & 35.286 & 57.324 & 48.732 & 70.429 & 37.571 \\
GE-3 (0.10) & 76.852 & 62.943 & 94.947 & 51.323 & 81.217 & 67.674 & 95.213 & 55.556 \\
GE-3 (0.20) & 61.477 & 51.406 & 83.062 & 39.865 & 64.966 & 54.083 & 85.366 & 42.432 \\
GE-3 (0.30) & 52.162 & 45.202 & 65.439 & 35.684 & 55.649 & 46.453 & 68.130 & 38.223 \\
\hline
\end{tabular}

For each of the four risk measures, that is, CoVaR, CoCaViaR, QL-CoVaR and QL-CoCaViaR, the table reports the percentages of times over the cross-section and over time in which the null hypothesis of the unconditional coverage test by Kupiec (1995) and the null hypothesis of the conditional coverage test by Christoffersen (1998) are not rejected at the $1 \%$ significance level. The tests are implemented during the periods of distress state of the conditioning financial companies. We detect the distress state of the financial companies using the hit function (13) for GE-1; GE-2 is obtained by replacing $\widehat{Q}_{\tau}\left(x_{i, t}\right)$ with $\widehat{q}_{\tau}\left(x_{i}\right)$ in (13), whereas we compute the hit function (14) at 3 different threshold values ths $-0.10(\mathrm{GE}-3(0.10)), 0.20(\mathrm{GE}-3(0.20))$ and $0.30(\mathrm{GE}-3(0.30))$. We implement the tests conditional to the fact that we have at least 50 days in which a given financial company is in distress, that is, 50 ones for each hit series of the company. CoVaR, CoCaViaR, QL-CoVaR and QL-CoCaViaR are computed by setting $\theta=\tau=0.05$ and $h=0.15$.

So far, we did not take into account the distress state of the conditioning companies. In contrast, Table 7 reports the results we obtain by conditioning the system's hit function to the distress state of each financial company. We detect the distress state of the individual companies by using different hit functions for $x_{i, t}$. The percentages of non-rejections we have when using (13) are given in GE-1, whereas GE-2 is obtained by replacing $\widehat{Q}_{\tau}\left(x_{i, t}\right)$ with $\widehat{q}_{\tau}\left(x_{i}\right)$ in (13). We also compute (14) at three different threshold values $\nu$ for a sensitivity analysis $-\nu=0.10$ in GE-3 (0.10), $\nu=0.20$ in GE-3 (0.20) and $\nu=0.30$ in GE-3 (0.30). For each of the hit functions (13)-with either $\widehat{Q}_{\tau}\left(x_{i, t}\right)$ or $\widehat{q}_{\tau}\left(x_{i}\right)$-and (14), we implement the unconditional and the conditional coverage tests for $y_{t}$ conditional to the fact that we have at least 50 days in which $H_{t, 0.5,0.5}^{i}=1$; that is, at least 50 days in which the $i$-th company is in distress. In general, $Q L-C o V a R_{t, 0.05,0.05}^{(i)}$ overperforms the other risk measures. Notably, 
the percentages of non-rejections for the quantile-located measures are greater in GE- 3 . This is due to the fact that the hit function of the conditioning companies in (14) is more consistent with the method we use to estimate both $Q L-C o V a R_{t, 0.05,0.05}^{(i)}$ and $Q L-C o C a V i a R_{t, 0.05,0.05}^{(i)}$.

Table 8: Average loss in different periods

\begin{tabular}{c|cccc}
\hline PERIOD & CoVaR & CoCaViaR & QL-CoVaR & QL-CoCaViaR \\
\hline $01 / 01 / 2004-02 / 07 / 2004$ & 4.972 & 7.075 & 4.977 & 7.339 \\
$01 / 07 / 2008-31 / 12 / 2008$ & 10.063 & 9.653 & 9.344 & 9.762 \\
$01 / 01 / 2015-03 / 07 / 2015$ & 4.297 & 4.213 & 3.740 & 4.611 \\
\hline
\end{tabular}

The table reports the average losses generated by the four risk measures-CoVaR, CoCaViaR, QL-CoVaR and QL-CoCaViaR - in three different periods: 01/01/2004-02/07/2004, 01/07/2008—31/12/2008 and $01 / 01 / 2015-03 / 07 / 2015$. The four risk measures are computed by setting $\theta=\tau=0.05$ and $h=0.15$.

We report in Table 8 the average losses generated by the competing risk measures, that we estimate from (16). We compute these losses for 3 different periods having the same size of 132 observations, that is, the pre-subprime crisis period $(01 / 01 / 2004-02 / 07 / 2004)$, the subprime crisis period $(01 / 07 / 2008$ - 31/12/2008) and the post-subprime crisis period $(01 / 01 / 2015-03 / 07 / 2015)$. As expected, the losses are smaller during tranquil periods and considerably increase during the subprime crisis. On average, the CoVaR overperforms the other measures in the interval 01/01/2004-02/07/2004, whereas the QL-CoVaR provides the lowest loss in the remaining periods. Interestingly, the distance between $C o V a R_{t, 0.05,0.05}^{(i)}$ and $Q L-C o V a R_{t, 0.05,0.05}^{(i)}$ increases in periods of financial distress, such as the days in the interval $01 / 07 / 2008-31 / 12 / 2008$.

We also test whether the losses defined in (16) generated by the four risk measures are statistically different. For this purpose, we implement the test proposed by Diebold and Mariano (2002) at the $5 \%$ significance level. For each of the three panels in Table 9, which respectively refer to the periods 01/01/2004-02/07/2004,01/07/2008 - 31/12/2008 and 01/01/2015 - 03/07/2015, we report the percentages of times over the cross-section in which the risk measures ordered by row outperform, in a statistically significant way, the risk measures ordered by column. For instance, the CoVaR outperforms the CoCaViaR in $21.62 \%$ of the cases during the period $01 / 01 / 2004-02 / 07 / 2004$, whereas the CoCaViaR outperforms the CoVaR in $1.01 \%$ of the cases in the same period. When considering net values (i.e., the percentage of times in which the $i$-th measure outperforms the $j$-th measure minus the percentage of times in which the former is outperformed by the latter, for $i \neq j$ ), it is interesting to observe that the quantile-located measures (QL-CoVaR and $\mathrm{QL}-\mathrm{CoCaViaR}$ ) record the best results during the crisis period. Indeed, the gap between the quantile-located measures and the other competitors is positive in the period $01 / 07 / 2008-31 / 12 / 2008$ and tends to be greater with respect to the pre- and the post-subprime crisis periods. 
Table 9: Difference in the performance statistically validated

\begin{tabular}{ccccc}
\hline & \multicolumn{4}{c}{$01 / 01 / 2004-02 / 07 / 2004$} \\
\hline & CoVaR & CoCaViaR & QL-CoVaR & QL-CoCaViaR \\
\cline { 2 - 4 } CoVaR & 0.000 & 21.622 & 11.824 & 31.757 \\
CoCaViaR & 1.014 & 0.000 & 3.378 & 18.243 \\
QL-CoVaR & 9.797 & 29.054 & 0.000 & 0.392 \\
QL-CoCaViaR & 1.689 & 15.541 & 1.014 & QL-CoCaViaR \\
\hline & & $01 / 07 / 2008-31 / 12 / 2008$ & 5.802 \\
CoVaR & CoVaR & CoCaViaR & QL-CoVaR & 8.215 \\
CoCaViaR & 0.000 & 6.826 & 3.754 & 0.000 \\
QL-CoVaR & 7.850 & 0.000 & 4.778 & 0.000 \\
QL-CoCaViaR & 22.867 & 14.334 & 2.389 & QL-CoCaViaR \\
\hline & 9.215 & 11.263 & 9.091 \\
CoVaR & & $01 / 01 / 2015-03 / 07 / 2015$ & 8.418 \\
CoCaViaR & CoVaR & CoCaViaR & QL-CoVaR & 9.764 \\
QL-CoVaR & 0.000 & 7.071 & 3.030 & 0.000 \\
QL-CoCaViaR & 2.694 & 0.000 & 4.040 & 0.000 \\
\hline
\end{tabular}

For each of the three periods: 01/01/2004-02/07/2004, 01/07/2008 -31/12/2008 and 01/01/2015-03/07/2015, the table reports the percentages of times over the cross-section in which the risk measure in the $i$-th row overperforms the risk measure in the $j$-th column for each of 3 panels, for $i, j=1, \ldots, 4$. The different performances of the four risk measures, that is, CoVaR, CoCaViaR, QL-CoVaR and QL-CoCaViaR, are validated by using the Diebold and Mariano (2002) test at the 5\% significance level. The four risk measures are computed by setting $\theta=\tau=0.05$ and $h=0.15$.

\subsection{Ex-ante identification of systemically important financial companies}

We now backtest the $\Delta$ Risk measures by using the methods described in Section 2.5. We use the same empirical set-up described in Section 4.3 and compute the $\Delta$ Risk measures by setting $\theta=\tau=0.05$, $h=0.15$ and $w s=300$. We focus on the subprime crisis and, following Girardi and Ergun (2013), use the interval June 1, 2006-May 31, 2007 as the training period (which corresponds to the pre-crisis period). This range includes 261 daily observations for each $\Delta$ Risk measure. We also define the testing or target period to be the crisis period, starting from June 1, 2007. As a robustness check, we consider four different dates to conclude the crisis period: May 30, 2008 (to have the same dimensionality of the pre-crisis period, that is, 261 daily observations), September 30, 2008, December 31, 2008 and February 27, 2009 (to include, at different time points, the effects of the collapse of Lehman Brothers). We focus on the companies for which we have no missing observations in both the pre- and the crisis periods. This ensures a proper comparison between ex-ante and ex-post evaluations. Given that we have four different target periods, the number of companies is not constant, and ranges from $N=348$ (when May 30, 2008 is the concluding date) to $N=332$ (when February 27, 2009 is the concluding date). Finally, we compute the dollar-valued $\Delta$ Risk measures and the changes in the companies market values, as discussed in Section 2.5. We first analyze the correlations between ex-ante estimates and ex-post realizations.

Table 10 reports the correlations computed for four different concluding dates of the subprime 
Table 10: Correlation between ex-ante estimates and ex-post realizations

\begin{tabular}{c|cccc}
\hline End of the crisis & $\Delta$ CoVaR & $\Delta$ CoCaViaR & $\Delta$ QL-CoVaR & $\Delta$ QL-CoCaViaR \\
\hline \multicolumn{5}{c}{ UNCONDITIONAL CORRELATIONS } \\
\hline May 30, 2008 & 84.007 & 83.257 & 86.029 & 87.473 \\
September 30, 2008 & 60.371 & 57.983 & 65.648 & 66.174 \\
December 31, 2008 & 83.024 & 81.534 & 86.904 & 87.587 \\
February 27, 2009 & 91.830 & 90.837 & 94.254 & 86.034 \\
\hline \multicolumn{5}{c}{ CONDITIONAL CORRELATIONS } \\
\hline May 30, 2008 & 81.613 & 80.550 & 60.228 \\
September 30, 2008 & 52.735 & 49.477 & 85.220 & 83.734 \\
December 31, 2008 & 77.094 & 74.912 & 83.401 & 93.044 \\
February 27, 2009 & 88.139 & 86.635 & 92.113 & \\
\hline
\end{tabular}

The top panel of the table reports the correlations between the average values of the pre-crisis $\$ \Delta$ Risk measures and the average changes in financial companies market values during the subprime crisis. We focus on the companies for which we have no missing observations from June 1, 2006 to the concluding date given in the first column of the table. In the bottom panel we report the correlations conditional to the fact that they are computed on the top-ranked systemic important financial companies in both the pre- and the crisis period.

crisis. Table 10 includes both unconditional (i.e., computed over the full set of companies) and conditional correlations (i.e., computed on the most risky companies that belong to the first decile of both the ex-ante and the ex-post ranking). In general, the four competing $\Delta$ Risk measures provide high correlations. Notably, the $\Delta \mathrm{QL}-\mathrm{CoCaViaR}$ records the best performance, providing the highest unconditional and conditional correlations. It is interesting to observe that the gap between the $\triangle \mathrm{QL}-\mathrm{CoCaViaR}$ and the $\triangle \mathrm{CoVaR}$ increases when moving from the unconditional to the conditional correlations, that is, when focusing on the top-ranked systemic important financial companies. The $\triangle \mathrm{QL}-\mathrm{CoVaR}$ records the best second performance.

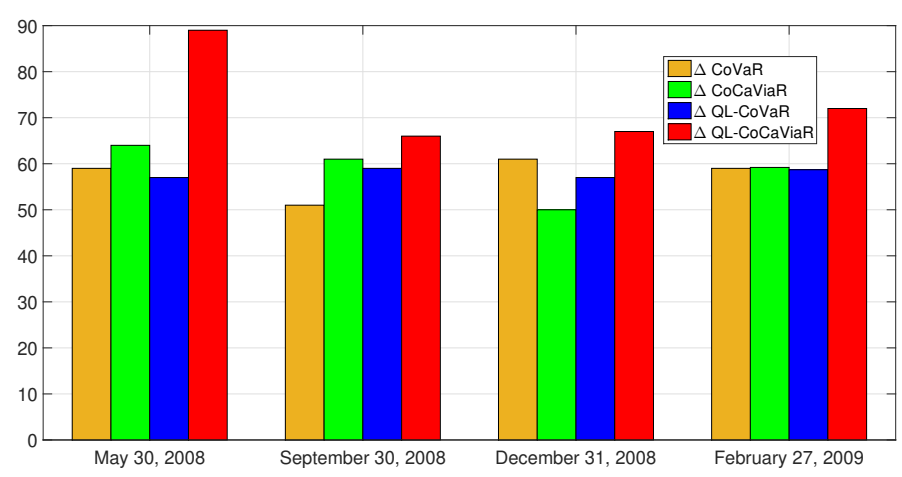

Figure 3: $C R$ ratio (\%) generated by the competing $\Delta$ Risk measures $-\Delta$ CoVaR, $\Delta$ CoCaViaR, $\Delta Q L-C o V a R$ and $\triangle \mathrm{QL}-\mathrm{CoCaViaR}$ - at different concluding dates of the crisis period, that is, May 30, 2008, September 30, 2008, December 31, 2008 and February 27, 2009.

As a second backtesting method, we compute the $C R$ ratio described in Section 2.5. We display the results in Figure 3. We note that the $\Delta \mathrm{QL}-\mathrm{CoCaViaR}$ systematically produces the highest $C R$ ratio, dominating the competing rules. Moreover, the $\triangle \mathrm{CoCaViaR}$ records the second best performance in three out of four cases. As a robustness check, we also compute the critical ratio $C R$ as the proportion of companies selected by the LASSO model (i.e., ex-post) that, at the same time, are also classified in 
the first three deciles of the ex-ante ranking. In doing so, we slightly relax the definition of systemically important company in the ex-ante evaluation. We check that the critical ratio $C R$ increases with this new definition for all the $\Delta$ Risk measures (see Figure S6 in the supplementary material). Still, the $\Delta \mathrm{QL}-\mathrm{CoCaViaR}$ systematically dominates the competing $\Delta$ Risk measures, with an average $C R$ ratio equal to 0.95 . The $\Delta$ CoCaViaR still records the best second performance with an average $C R$ ratio equal to 0.86 , whereas the $\Delta \mathrm{CoVaR}$ produces, on average, a $C R$ ratio equal to 0.83 .

\section{$5 \quad \Delta$ Risk measures and systemically important financial institutions}

Every year the the Financial Stability Board (FSB) classifies some financial institutions as as global systemically important (G-SIFIs). In particular, the FSB identifies the G-SIFIs by aggregating several indicators that convey information about different features of a company, such as size, cross-jurisdictional activity, interconnectedness, substitutability and complexity. ${ }^{21}$ In contrast, we assess the systemic relevance of each company in our dataset by means of $\Delta$ Risk measures, that build on stock prices and some control variables related to bond, equity and real-estate markets. Therefore, it is important to investigate whether there exists a relation between these two different approaches and thereby our proposal could become an effective tool for risk monitoring for reggulations.

As our dataset includes US companies, while the G-SIFIs identified by the FSB have different jurisdictions, we consider only the US jurisdiction in the period 2011 - 2015, due to the data availability and check that the composition of the US G-SIFIs does not change during the period 2011-2015. Then, the US G-SIBs are the following: Bank of America (BAC), Bank of New York Mellon (BK), Citigroup (C), Goldman Sachs (GS), JP Morgan Chase (JPM), Morgan Stanley (MS), State Street (STT) and Wells Fargo (WFC). The US G-SIIs are the following: American International Group (AIG), MetLife (MET) and Prudential Financial (PRU).

We estimate the competing $\Delta$ Risk measures from the returns observed during the period $2011-2015$ and focus on 461 companies for which we have data in the entire period. Following the empirical set-up used in Sections 4.3-4.4, we set $\theta=\tau=0.05$ and $h=0.15$ and then compute the mean of the $\Delta$ CoVaR for each of the 461 companies during the period 2011-2015, denoted as $\overline{\Delta C o V a R_{j}}$, for $j=1, \ldots, 461$. We then build the empirical distribution function of the series $\overline{\Delta \mathbf{C o V a R}}=$ $\left[\overline{\Delta C o V a R_{1}} \ldots \overline{\Delta C o V a R_{461}}\right]$, obtaining the quantile level of the $j$-th company, that is, $\alpha_{j, \Delta C o V a R}=$ $Q_{\alpha_{j}}^{-1}(\overline{\Delta \mathbf{C o V a R}}) \in(0,1]$, for $j=1, \ldots, 461$. For instance, $\alpha_{j, \Delta \mathrm{CoVaR}}=1 / 461$ if the $j$-th company has the lowest (negative) value of $\overline{\Delta C o V a R_{j}}$, that is, the company with the strongest systemic impact. Likewise, we compute the quantile levels $\alpha_{j, \Delta Q L-C o V a R}$ when focusing on the $\triangle \mathrm{QL}-\mathrm{CoCaViaR}$. Figure 4 displays the scatter plot of the series $\alpha_{j, \Delta C o V a R}$ and $\alpha_{j, \Delta Q L-C o V a R}$. We can see from Figure 4 that $\alpha_{j, \Delta C o V a R}$ and $\alpha_{j, \Delta Q L-C o V a R}$ are positively correlated, with a linear correlation coefficient of 0.9088 .

\footnotetext{
${ }^{21}$ See the website http://www.fsb.org for additional details.
} 
The US G-SIFIs are positioned on the bottom left-hand corner of the scatter plot, that includes the companies with the greatest systemic impact according to both $\Delta$ CoVaR and $\Delta \mathrm{QL}-\mathrm{CoCaViaR}$. We notice that when using the $\Delta \mathrm{QL}-\mathrm{CoCaViaR}$, ten out of eleven US G-SIFIs are within the first decile, whereas only the quantile level of STT is slightly greater than the first decile CHECK FIGURE AS DOTS ARE NOT VISIBLE. In contrast, BAC, GS and AIG are clearly distant from the first decile when using the $\Delta$ CoVaR. Thereby, despite using promptly available and limited information compared to the methodology adopted by the FSB, the $\Delta \mathrm{QL}-\mathrm{CoCaViaR}$ is still capable of correctly identifying the US G-SIFIs as among the most risky companies. Furthermore, we also check that the most general specification we propose $-\Delta \mathrm{QL}-\mathrm{CoCaViaR}$ - outperforms the two sub-models- $\Delta \mathrm{CoCaViaR}$ and $\Delta$ QL-CoVaR - in identifying the US G-SIFIs. In turn, these two sub-models outperform the $\Delta \mathrm{CoVaR}^{22}$

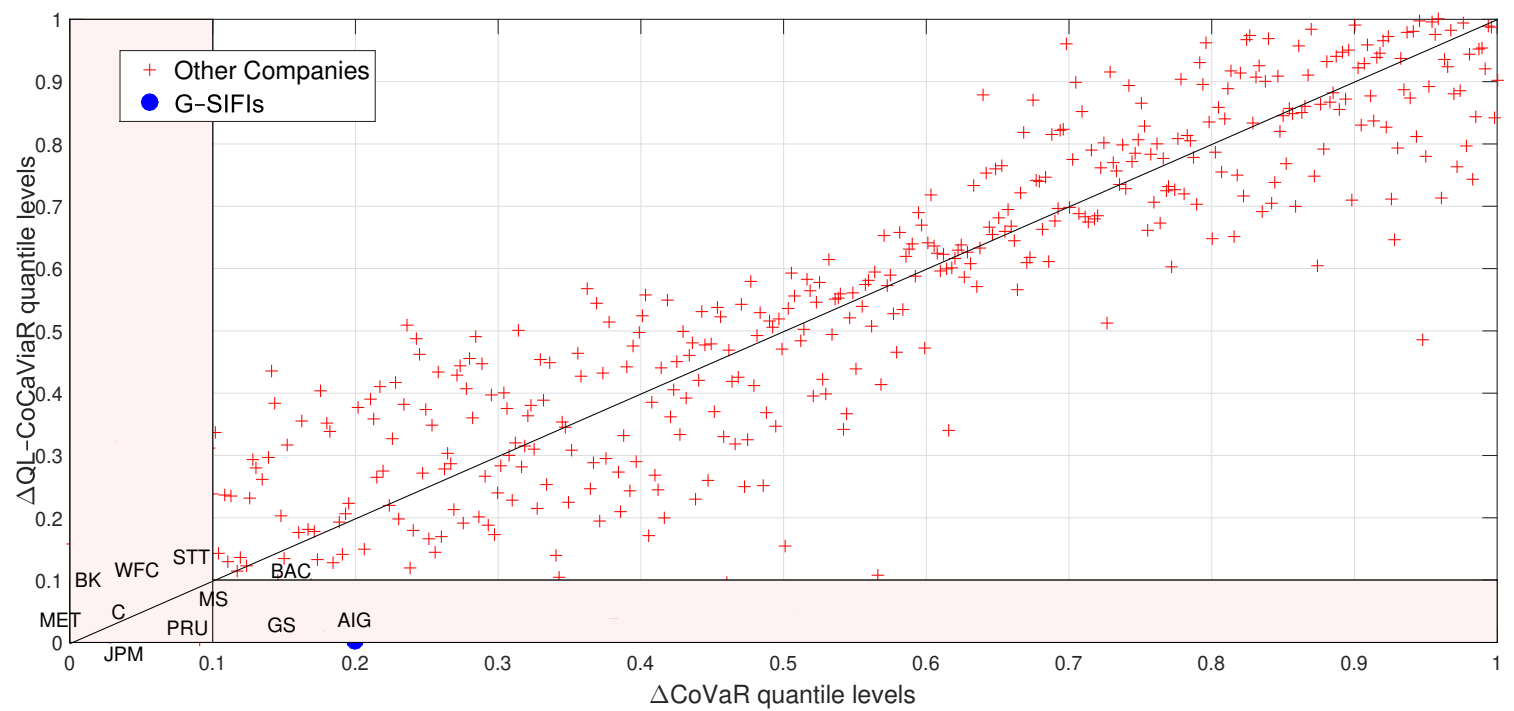

Figure 4: The figure displays the quantile levels of the US G-SIFIs identified by the FSB-American International Group (AIG), Bank of America (BAC), Bank of New York Mellon (BK), Citigroup (C), Goldman Sachs (GS), JP Morgan Chase (JPM), MetLife (MET), Morgan Stanley (MS), Prudential Financial (PRU), State Street (STT) and Wells Fargo (WFC) - within the rankings obtained by using the $\Delta \mathrm{CoVaR}$ and the $\Delta \mathrm{QL}-\mathrm{CoCaViaR}$, respectively.

\section{Concluding remarks}

We extend the CoVaR introduced by Adrian and Brunnermeier (2016), taking into account the persistence of quantiles over time (CoCaViaR), quantile-located relationships (QL-CoVaR) and their combination (QL-CoCaViaR). An extensive empirical analysis based on a large dataset including US banks and insurance companies highlights the relevance of the new methods we introduce. First, we check that the CoCaViaR is more conservative than the other risk measures. Indeed, the CaViaR's components (Engle and Manganelli, 2004) absorb, in part, the relations between the financial system

\footnotetext{
${ }^{22}$ We do not report here the scatter plots for $\Delta \mathrm{CoCaViaR}$ and $\Delta \mathrm{QL}-\mathrm{CoVaR}$ for the sake of space. These scatter plots are available upon request.
} 
and the individual companies, lowering the contribution of the firms to the systemic risk. Second, the relationships between the financial system and the individual companies become stronger when linking the left tails of their returns' distributions, that is, when accentuating the distress degree in their connections (quantile-located effects). As a result, both QL-CoVaR and QL-CoCaViaR capture stronger structural relationships between the market and the individual companies during crisis periods.

We use various backtesting methods for evaluating the predictive accuracy of the competing risk measures. In addition to well-known methods in the literature, we develop novel approaches to deal with the quantile-location. In particular, the empirical evidence shows that the quantile-located relationships are particularly useful in improving the predictive accuracy during stressed periods, making it a relevant tool for regulators to either manage or prevent the effects of extreme events.

Here, we focus on the relations between the financial system and the individual companies when the former is in distress. However, in order to shed further light on the capabilities of our method, it would be interesting to check how the results change when the focus is on the upper tail of the system. Besides, it would be interesting to compare our approach with other statistical methods, that can differ from the quantile regression, proposed in the literature to estimate the CoVaR. An example is the GARCH model used by Girardi and Ergun (2013). We include these further analyses in our research agenda.

\section{References}

Acharya, V., L. Pedersen, T. Philippon, and M. Richardson (2017). Measuring systemic risk. The Review of Financial Studies 30, 2-47.

Adrian, T. and M. K. Brunnermeier (2016). CoVaR. American Economic Review 106, 1705-1741.

Allen, F. and D. Gale (2000). Financial contagion. Journal of Political Economy 108, 1-33.

Bekaert, G., E. Engstrom, and A. Ermolov (2015). Bad environments, good environments: A nongaussian asymmetric volatility model. Journal of Econometrics 186, 258-275.

Benoit, S., J. Colliard, C. Hurlin, and C. Pérignon (2017). Where the risks lie: A survey on systemic risk. Review of Finance 21, 109-152.

Bernal, O., J. Gnabo, and G. Guilmin (2014). Assessing the contribution of banks, insurance and other financial services to systemic risk. Journal of Banking \& Finance 47, 270-287.

Bernardi, M., G. Gayraud, and L. Petrella (2015). Bayesian tail risk interdependence using quantile regression. Bayesian Analysis 10, 553-603.

Bernardi, M., A. Maruotti, and L. Petrella (2017). Multiple risk measures for multivariate dynamic heavy-tailed models. Journal of Empirical Finance 43, 1-32. 
Billio, M., M. Getmansky, A. Lo, and L. Pelizzon (2012). Econometric measures of systemic risk in the finance and insurance sectors. Journal of Financial Economics 104, 535-559.

Bondell, H. D., B. J. Reich, and H. Wang (2010). Non-crossing quantile regression curve estimation. Biometrika 97, 825-838.

Brownlees, C. and R. Engle (2017). SRISK: A conditional capital shortfall measure of systemic risk. The Review of Financial Studies 30, 48-79.

Brunnermeier, M. and L. Pedersen (2009). Market liquidity and funding liquidity. The Review of Financial Studies 22, 2201-2238.

Calomiris, C. and C. Kahn (1991). The role of demandable debt in structuring optimal banking arrangements. American Economic Review 81, 497-513.

Caporin, M. (2008). Evaluating Value-at-Risk measures in the presence of long memory conditional volatility. Journal of Risk 10, 79-110.

Castro, C. and S. Ferrari (2014). Measuring and testing for the systemically important financial institutions. Journal of Empirical Finance 25, 1-14.

Chen, Y. (1999). Banking panics: The role of the first-come, first-served rule and information externalities. Journal of Political Economy 107, 946-968.

Christoffersen, P. (1998). Evaluating interval forecasts. International Economic Review 39, 841-862.

Cont, R. (2001). Empirical properties of asset returns: stylized facts and statistical issues. Quantitative Finance 1(2), 223-236.

Dasgupta, A. (2004). Financial contagion through capital connections: A model of the origins and spread of bank panics. Journal of the European Economic Association 2, 1049-1084.

Diamond, D. and P. Dybvig (1983). Bank runs, deposit insurance, and liquidity. Journal of Political Economy 91, 401-419.

Diebold, F. and R. Mariano (2002). Comparing predictive accuracy. Journal of Business \& Economic Statistics 20, 134-144.

Duffie, D. and H. Zhu (2011). Does a central clearing counterparty reduce counterparty risk? Review of Asset Pricing Studies 1, 74-95.

Efron, B. (1979). Bootstrap methods: Another look at the jackknife. The Annals of Statistics 7, 1-26.

Engle, R. F. and S. Manganelli (2004). CAViaR: Conditional autoregressive Value at Risk by regression quantiles. Journal of Business \&3 Economic Statistics 22, 367-381. 
Freixas, X. and B. Parigi (1998). Contagion and efficiency in gross and net interbank payment systems. Journal of Financial Intermediation 7, 3-31.

Freixas, X., B. Parigi, and J. Rochet (2000). Systemic risk, interbank relations, and liquidity provision by the central bank. Journal of Money, Credit and Banking 32, 611-638.

Girardi, G. and A. T. Ergun (2013). Systemic risk measurement: Multivariate GARCH estimation of CoVaR. Journal of Banking \& Finance 37, 3169-3180.

Kocherginsky, M. (2003). Extensions of the Markov chain marginal bootstrap. PhD Thesis, University of Illinois Urbana-Champaign.

Koenker, R. (2005). Quantile regression. Number 38. Cambridge university press.

Koenker, R. and G. Bassett (1978). Regression quantiles. Econometrica 46(1), 33-50.

Koenker, R. and Z. Xiao (2004). Unit root quantile autoregression inference. Journal of the American Statistical Association 99, 775-787.

Kupiec, P. (1995). Techniques for verifying the accuracy of risk measurement models. Journal of Derivatives 3, 73-84.

Li, G., Y. Li, and C. L. Tsai (2015). Quantile correlations and quantile autoregressive modeling. Journal of the American Statistical Association 110, 246-261.

López-Espinosa, G., A. Moreno, A. Rubia, and L. Valderrama (2012). Short-term wholesale funding and systemic risk: A global covar approach. Journal of Banking E Finance 36, 3150-3162.

López-Espinosa, G., A. Moreno, A. Rubia, and L. Valderrama (2015). Systemic risk and asymmetric responses in the financial industry. Journal of Banking $\&$ Finance 58, 471-485.

Martin, A., D. Skeie, and E. V. Thadden (2014). Repo runs. The Review of Financial Studies 27, 957-989.

Qu, Z. (2008). Testing for structural change in regression quantiles. Journal of Econometrics 146, $170-184$.

Rochet, J. and J. Tirole (1996). Controlling risk in payment systems. Journal of Money, Credit and Banking 28, 832-862.

Rosengren, E. (2010). Asset bubbles and systemic risk. Technical report, Federal Reserve Bank of Boston.

Shleifer, A. and R. Vishny (1992). Liquidation values and debt capacity: A market equilibrium approach. Journal of Finance 47, 1343-1366. 
Sim, N. and H. Zhou (2015). Oil prices, us stock return, and the dependence between their quantiles. Journal of Banking 86 Finance 55, 1-8.

White, H., T. Kim, and S. Manganelli (2015). VAR for VaR: Measuring tail dependence using multivariate regression quantiles. Journal of Econometrics 18\%, 169-188.

Zou, H., T. Hastie, and R. Tibshirani (2006). Sparse principal component analysis. Journal of Computational and Graphical Statistics 15, 265-286. 


\section{Supplementary material}

\section{S1 Derivation of the $\triangle Q L-C o C a V i a R$ in Equation (11)}

$$
\begin{aligned}
\Delta Q L-C o C a V i a R_{t, \tau}^{(i)} & =Q L-C o C a V i a R_{t, \theta, \tau}^{(i)}-Q L-C o C a V i a R_{t, \theta, 1 / 2}^{(i)} \\
& =\widehat{\delta}_{\theta, \tau}^{(i)}+\widehat{\psi}_{1, \theta, \tau}^{(i)} \widehat{Q}_{\theta, \tau}^{(i)}\left(y_{t-1}\right)+\widehat{\psi}_{2, \theta, \tau}^{(i)}\left|y_{t-1}\right|+\widehat{\lambda}_{\theta, \tau}^{(i)} \widehat{Q}_{\tau}\left(x_{i, t}\right)+\widehat{\gamma}_{\theta, \tau}^{(i)} \mathbf{M}_{t-1}^{\prime} \\
& -\widehat{\delta}_{\theta, 1 / 2}^{(i)}-\widehat{\psi}_{1, \theta, 1 / 2}^{(i)} \widehat{Q}_{\theta, 1 / 2}^{(i)}\left(y_{t-1}\right)-\widehat{\psi}_{2, \theta, 1 / 2}^{(i)}\left|y_{t-1}\right|-\widehat{\lambda}_{\theta, 1 / 2}^{(i)} \widehat{Q}_{1 / 2}\left(x_{i, t}\right) \\
& -\widehat{\gamma}_{\theta, 1 / 2}^{(i)} \mathbf{M}_{t-1}^{\prime} \\
& =\widehat{\delta}_{\theta, \tau}^{(i)}+\widehat{\psi}_{1, \theta, \tau}^{(i)} \widehat{Q}_{\theta, \tau}^{(i)}\left(y_{t-1}\right)+\widehat{\psi}_{2, \theta, \tau}^{(i)}\left|y_{t-1}\right|+\widehat{\lambda}_{\theta, \tau}^{(i)} \widehat{Q}_{\tau}\left(x_{i, t}\right)+\widehat{\gamma}_{\theta, \tau}^{(i)} \mathbf{M}_{t-1}^{\prime} \\
& -\widehat{\delta}_{\theta, 1 / 2}^{(i)}-\widehat{\psi}_{1, \theta, 1 / 2}^{(i)} \widehat{Q}_{\theta, 1 / 2}^{(i)}\left(y_{t-1}\right)-\widehat{\psi}_{2, \theta, 1 / 2}^{(i)}\left|y_{t-1}\right|-\widehat{\lambda}_{\theta, 1 / 2}^{(i)} \widehat{Q}_{1 / 2}\left(x_{i, t}\right) \\
& -\widehat{\gamma}_{\theta, 1 / 2}^{(i)} \mathbf{M}_{t-1}^{\prime}+\widehat{\lambda}_{\theta, \tau}^{(i)} \widehat{Q}_{1 / 2}\left(x_{i, t}\right)-\widehat{\lambda}_{\theta, \tau}^{(i)} \widehat{Q}_{1 / 2}\left(x_{i, t}\right) \\
& +\widehat{\psi}_{1, \theta, 1 / 2}^{(i)} \widehat{Q}_{\theta, \tau}^{(i)}\left(y_{t-1}\right)-\widehat{\psi}_{1, \theta, 1 / 2}^{(i)} \widehat{Q}_{\theta, \tau}^{(i)}\left(y_{t-1}\right) \\
& =\left(\widehat{\delta}_{\theta, \tau}^{(i)}-\widehat{\delta}_{\theta, 1 / 2}^{(i)}\right)+\widehat{\lambda}_{\theta, \tau}^{(i)}\left[\widehat{Q}_{\tau}\left(x_{i, t}\right)-\widehat{Q}_{1 / 2}\left(x_{i, t}\right)\right]+\left(\widehat{\lambda}_{\theta, \tau}^{(i)}-\widehat{\lambda}_{\theta, 1 / 2}^{(i)}\right) \widehat{Q}_{1 / 2}\left(x_{i, t}\right) \\
& +\left(\widehat{\gamma}_{\theta, \tau}^{(i)}-\widehat{\gamma}_{\theta, 1 / 2}^{(i)}\right) \mathbf{M}_{t-1}^{\prime}+\left(\widehat{\psi}_{2, \theta, \tau}^{(i)}-\widehat{\psi}_{2, \theta, 1 / 2}^{(i)}\right)\left|y_{t-1}\right| \\
& +\left[\widehat{\psi}_{1, \theta, \tau}^{(i)}-\widehat{\psi}_{1, \theta, 1 / 2}^{(i)}\right] \widehat{Q}_{\theta, \tau}^{(i)}\left(y_{t-1}\right) \\
& +\widehat{\psi}_{1, \theta, 1 / 2}^{(i)}\left[\widehat{Q}_{\theta, \tau}^{(i)}\left(y_{t-1}\right)-\widehat{Q}_{\theta, 1 / 2}^{(i)}\left(y_{t-1}\right)\right]
\end{aligned}
$$

\section{S2 Additional details on the dataset}

Panel (a) of Figure S1 displays the number of banks and insurance companies present from October 10, 2000 to July 31, 2015. The number of present insurances is quite constant over time, with a mean value of 88. In contrast, the number of banks increases in the interval October 10, 2000-July 31, 2015, from 253 to 543. We analyze the monthly variations of the number of present companies in Panel (b) of Figure S1. The histograms reported here show that these variations are more volatile for banks (interquartile range of 3) than for insurances (interquartile range of 1). Moreover, the distribution we build from banks has a remarkable right tail (skewness index equal to 0.62). The median of these variations is equal to 1 (0) for banks (insurances). Then, banks have larger monthly increments.

We divide our dataset into 3 sub-periods (i.e., October 10, 2000-September 15, 2005 (the first), September 16, 2005-August 25, 2010 (the second) and August 26, 2010-July 31, 2015 (the third)) to provide a more accurate description. Here, different descriptive statistics are computed for banks and insurances separately. In particular, for each company for which we have at least 50 observations, we compute the following statistics: 5 -th and 95-th percentiles, median and interquartile range. The 


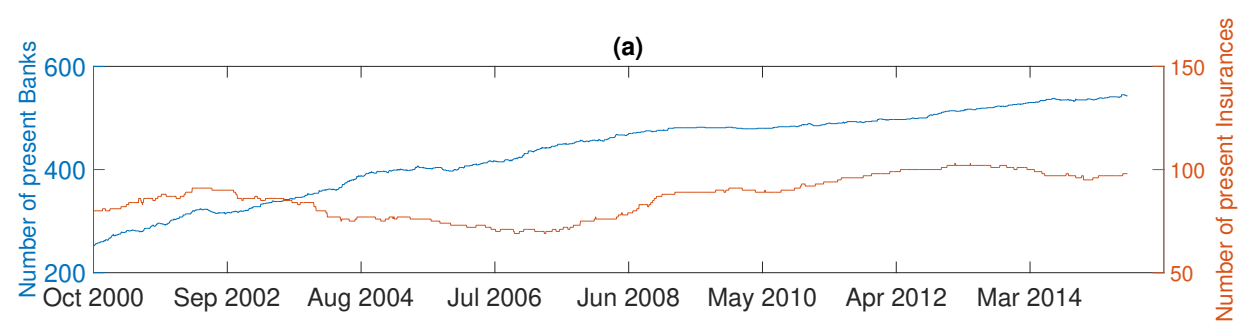

(b)

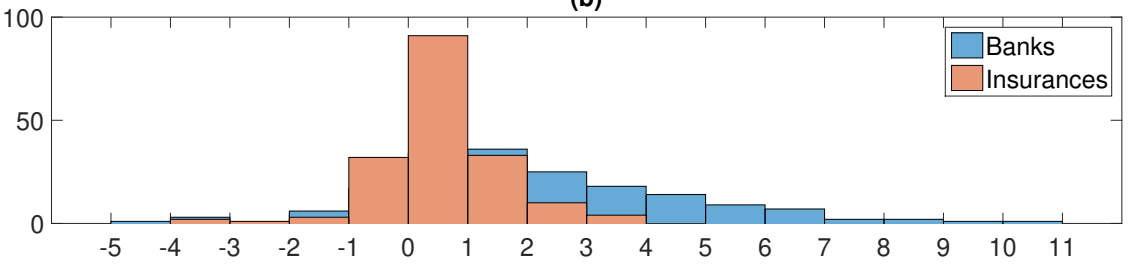

Figure S1: Panel (a) displays the number of banks and insurance companies present in our dataset for each day in the interval between October 10, 2000 and July 31, 2015. Panel (b) displays the histograms of the monthly variations of the number of present companies in the interval between October 10, 2000 and July 31, 2015, distinguishing banks from insurances.

Table S1: Descriptive statistics of daily financial returns

\begin{tabular}{c|cccc|cccc}
\hline & \multicolumn{4}{|c|}{ BANKS } & \multicolumn{4}{c}{ INSURANCES } \\
\hline PERIOD & 5P & MED & $95 \mathrm{P}$ & IQR & 5P & MED & 95P & IQR \\
\hline $10 / 10 / 2000-15 / 09 / 2005$ & -2.666 & 0.080 & 2.991 & 1.217 & -2.773 & 0.050 & 2.982 & 1.651 \\
$16 / 09 / 2005-25 / 08 / 2010$ & -4.285 & -0.028 & 4.157 & 1.640 & -3.533 & 0.005 & 3.490 & 1.829 \\
$26 / 08 / 2010-31 / 07 / 2015$ & -2.859 & 0.058 & 3.034 & 1.622 & -2.309 & 0.064 & 2.401 & 1.443 \\
\hline
\end{tabular}

We divide the dataset into 3 sub-periods: October 10, 2000-September 15, 2005 (first), September 16, 2005-August 25, 2010 (second), August 26, 2010-July 31, 2015 (third). For each institution for which we have at least 50 observations, we compute the following descriptive statistics: 5-th (5P, \%) and 95-th (95P, \%) percentiles, median (MED, \%) and interquartile range (IQR, \%). Table S1 reports the cross-sectional medians of these statistics.

cross-sectional medians of these statistics are then reported in Table S1. The 5 -th percentile and the median tend to be lower in the period between September 16, 2005 and August 25, 2010. The 95-th percentile and the interquartile range take greater values in the same interval. This highlights the worsening of the companies' performance in terms of profitability and risk during the subprime crisis.

\section{S3 Analysis of the risk measures' coefficients}

Table S2: Estimation of $Q_{\theta}^{(i)}\left(y_{t}\right)=\delta_{\theta}^{(i)}+\lambda_{\theta}^{(i)} x_{i, t}+\gamma_{\theta}^{(i)} f p c_{t-1}$

\begin{tabular}{c|ccccc|ccccc}
\hline & \multicolumn{3}{|c|}{$\theta=0.01$} & & \multicolumn{5}{c}{$\theta=0.05$} \\
\hline COEF & $5 \mathrm{P}$ & MED & $95 \mathrm{P}$ & IQR & PS & $5 \mathrm{P}$ & MED & $95 \mathrm{P}$ & IQR & PS \\
\hline$\delta_{\theta}$ & -0.042 & -0.031 & -0.021 & 0.012 & 99.903 & -0.027 & -0.019 & -0.014 & 0.007 & 99.612 \\
$\lambda_{\theta}$ & -0.033 & 0.117 & 0.536 & 0.236 & 45.146 & -0.006 & 0.112 & 0.561 & 0.276 & 57.087 \\
$100 \times \gamma_{\theta}$ & -0.295 & -0.197 & -0.074 & 0.107 & 88.447 & -0.202 & -0.128 & -0.079 & 0.064 & 95.243 \\
\hline
\end{tabular}

The table reports the summary statistics of the CoVaR's parameters estimated for the $N$ financial companies included in our dataset. The estimates are obtained using two quantile levels - $\theta$. In each panel, from left to right, we report the following descriptive statistics of the coefficients: the 5 -th percentile (5P), the median (MED), the 95-th percentile $(3 \mathrm{Q})$, the interquartile range (IQR) and the percentage of times, over the cross-section, in which they are statistically significant at the $5 \%$ confidence level (PS). 
Table S3: Estimation of $Q_{\theta}^{(i)}\left(y_{t}\right)=\delta_{\theta}^{(i)}+\psi_{1, \theta}^{(i)} Q_{\theta}^{(i)}\left(y_{t-1}\right)+\psi_{2, \theta}^{(i)}\left|y_{t-1}\right|+\lambda_{\theta}^{(i)} x_{i, t}+\gamma_{\theta}^{(i)} f p c_{t-1}$

\begin{tabular}{c|ccccc|ccccc}
\hline & \multicolumn{9}{|c|}{$\theta=0.01$} & \multicolumn{5}{c}{$\theta=0.05$} \\
\hline COEF & $5 \mathrm{P}$ & MED & $95 \mathrm{P}$ & IQR & PS & $5 \mathrm{P}$ & MED & $95 \mathrm{P}$ & IQR & PS \\
\hline$\delta_{\theta}$ & -0.042 & -0.022 & -0.001 & 0.020 & 78.155 & -0.027 & -0.014 & -0.001 & 0.015 & 83.689 \\
$\psi_{1, \theta}$ & -0.491 & 0.197 & 0.917 & 0.916 & 55.728 & -0.616 & 0.142 & 0.895 & 0.815 & 52.816 \\
$\psi_{2, \theta}$ & -0.321 & -0.132 & 0.147 & 0.203 & 38.932 & -0.211 & -0.100 & 0.067 & 0.134 & 42.427 \\
$\lambda_{\theta}$ & -3.711 & 0.101 & 0.510 & 0.248 & 78.447 & -0.005 & 0.097 & 0.557 & 0.280 & 76.505 \\
$100 \times \gamma_{\theta}$ & -0.251 & -0.119 & -0.003 & 0.161 & 70.194 & -0.159 & -0.083 & -0.005 & 0.096 & 82.524 \\
\hline
\end{tabular}

The table reports the summary statistics of the CoCaViaR's parameters for the $N$ financial companies included in our dataset. The estimates are obtained using two quantile levels - $\theta$. In each panel, from left to right, we report the following descriptive statistics of the coefficients: the 5 -th percentile (5P), the median (MED), the 95-th percentile (95P), the interquartile range (IQR) and the percentage of times, over the cross-section, in which they are statistically significant at the $5 \%$ confidence level (PS).

Table $S 2$ reports the statistics of the CoVaR's coefficients, obtained by estimating $Q_{\theta}^{(i)}\left(y_{t}\right)=$ $\delta_{\theta}^{(i)}+\lambda_{\theta}^{(i)} x_{i, t}+\gamma_{\theta}^{(i)} f p c_{t-1}$, for $i=1, \ldots, N$. On average, the individual companies have a positive impact on $Q_{\theta}^{(i)}\left(y_{t}\right)$. Indeed, the medians of $\widehat{\lambda}_{0.01}^{(i)}$ and $\widehat{\lambda}_{0.05}^{(i)}$ - the coefficients that monitor the impact of a financial company on the market risk - are similar, being equal to 0.117 and 0.112 , respectively. $\hat{\lambda}_{0.05}^{(i)}$ is slightly more volatile than $\hat{\lambda}_{0.01}^{(i)}$. Indeed, the former (the latter) has an interquartile range of 0.276 (0.236). Both the 5-th and the 95-th percentiles are larger for $\hat{\lambda}_{0.05}^{(i)}(-0.006$ and 0.561 , respectively) than for $\hat{\lambda}_{0.01}^{(i)}\left(-0.033\right.$ and 0.536 , respectively). $\hat{\lambda}_{0.05}^{(i)}$ records a larger number of times in which it is statistically significant at the $5 \%$ confidence level with respect to $\hat{\lambda}_{0.01}^{(i)}(57 \%$ versus $45 \%)$. Overall, we do not observe relevant differences in the impact of financial companies on the system when comparing $\theta=0.05$ with $\theta=0.01$. When focusing on the impact of the control variables, as expressed by their first principal component $\left(f p c_{t-1}\right)$, we again observe similarities when comparing $\theta=0.01$ with $\theta=0.05$. $\widehat{\gamma}_{\theta}^{(i)}$ is statistically significant for the majority of companies, highlighting a relevant role of the control variables in the evaluation of the market risk.

Table S3 includes the descriptive statistics of the CoCaViaR's coefficients estimated from $Q_{\theta}^{(i)}\left(y_{t}\right)=$ $\delta_{\theta}^{(i)}+\psi_{1, \theta}^{(i)} Q_{\theta}^{(i)}\left(y_{t-1}\right)+\psi_{2, \theta}^{(i)}\left|y_{t-1}\right|+\lambda_{\theta}^{(i)} x_{i, t}+\gamma_{\theta}^{(i)} f p c_{t-1}$. On average, the lagged quantile of the financial system- $Q_{\theta}^{(i)}\left(y_{t-1}\right)$ - has a positive impact on its current value $-Q_{\theta}^{(i)}\left(y_{t}\right)$. The relationships are stronger when setting $\theta=0.01$ (the median of $\widehat{\psi}_{1,0.01}^{(i)}$ is equal to 0.197) than $\theta=0.05$ (the median of $\widehat{\psi}_{1,0.05}^{(i)}$ is equal to 0.142$)$. As a result, the persistence in quantiles is more relevant in more extreme market states. $\widehat{\psi}_{1,0.01}^{(i)}$ and $\widehat{\psi}_{1,0.05}^{(i)}$ take moderate values, lower than the levels typically observed in the CaViaR model by Engle and Manganelli (2004). Therefore, the relationships between the financial system and the individual companies absorb, in part, the effects typically exerted by the CaViaR components. $\widehat{\psi}_{1,0.01}^{(i)}$ is slightly more volatile than $\widehat{\psi}_{1,0.05}^{(i)}$, as the interquartile range of the former (the latter) is equal to $0.916(0.816)$. Further, $\widehat{\psi}_{1,0.01}^{(i)}$ and $\widehat{\psi}_{1,0.05}^{(i)}$ are statistically significant at the $5 \%$ level for the majority of financial companies.

On average, $\left|y_{t-1}\right|$, the symmetric innovation term of the CoCaViaR, has a negative impact on $Q_{\theta}^{(i)}\left(y_{t}\right)$ : the greater the absolute values of the lagged returns of the system, the higher the extreme 
Table S4: Robustness check on the CoCaViaR model

\begin{tabular}{c|ccccc|ccccc}
\hline & \multicolumn{7}{c|}{$\theta=0.01$} & \multicolumn{5}{c}{$\theta=0.05$} \\
\hline COEF & $5 \mathrm{P}$ & MED & $95 \mathrm{P}$ & IQR & PS & $5 \mathrm{P}$ & MED & $95 \mathrm{P}$ & IQR & PS \\
\hline \multicolumn{8}{c}{ (a) Estimation of $Q_{\theta}^{(i)}\left(y_{t}\right)=\delta_{\theta}^{(i)}+\psi_{1, \theta}^{(i)} Q_{\theta}^{(i)}\left(y_{t-1}\right)+\psi_{2, \theta}^{(i)}\left|y_{t-1}\right|+\lambda_{\theta}^{(i)} x_{i, t}$} \\
\hline$\delta_{\theta}$ & -0.016 & -0.001 & 0.000 & 0.001 & 80.012 & -0.009 & -0.001 & 0.000 & 0.002 & 81.293 \\
$\psi_{1, \theta}$ & 0.308 & 0.909 & 0.993 & 0.078 & 59.135 & 0.177 & 0.910 & 0.975 & 0.198 & 61.121 \\
$\psi_{2, \theta}$ & -0.602 & -0.236 & 0.092 & 0.132 & 39.652 & -0.521 & -0.168 & 0.033 & 0.147 & 45.382 \\
$\lambda_{\theta}$ & -0.018 & 0.072 & 0.408 & 0.131 & 77.824 & -0.005 & 0.057 & 0.561 & 0.156 & 75.106 \\
\hline \multicolumn{10}{c}{ (b) Estimation of $Q_{\theta}^{(i)}\left(y_{t}\right)=\delta_{\theta}^{(i)}+\psi_{1, \theta}^{(i)} Q_{\theta}^{(i)}\left(y_{t-1}\right)+\lambda_{\theta}^{(i)} x_{i, t}+\gamma_{\theta}^{(i)} f p c_{t-1}$} \\
\hline$\delta_{\theta}$ & -0.045 & -0.026 & -0.009 & 0.013 & 79.104 & -0.027 & -0.017 & -0.009 & 0.006 & 82.891 \\
$\psi_{1, \theta}$ & -0.518 & 0.094 & 0.719 & 0.530 & 53.992 & -0.602 & 0.067 & 0.576 & 0.405 & 47.892 \\
$\lambda_{\theta}$ & -0.040 & 0.122 & 0.505 & 0.244 & 80.222 & -0.009 & 0.106 & 0.567 & 0.279 & 79.815 \\
$100 \times \gamma_{\theta}$ & -0.269 & -0.165 & -0.046 & 0.082 & 76.651 & -0.168 & -0.112 & -0.053 & 0.041 & 84.626 \\
\hline
\end{tabular}

The table reports summary statistics computed on the coefficients of the CoCaViaR model without the presence of either $f p c_{t-1}$ (panel (a)) or $\left|y_{t-1}\right|$ (panel (b)) for the $N$ financial companies included in our dataset. The estimates are obtained by setting $\theta=\{0.01,0.05\}$. In each panel, from left to right, we report the following descriptive statistics of the coefficients: the 5 -th percentile (5P), the median (MED), the 95-th percentile (95P), the interquartile range (IQR) and the percentage of times, over the cross-section, in which they are statistically significant at the $5 \%$ confidence level (PS).

Table S5: Estimation of $Q_{\theta, \tau}^{(i)}\left(y_{t}\right)=\delta_{\theta, \tau}^{(i)}+\lambda_{\theta, \tau}^{(i)} x_{i, t}+\gamma_{\theta, \tau}^{(i)} f p c_{t-1}$ and $Q_{\theta, 1 / 2}^{(i)}\left(y_{t}\right)=\delta_{\theta, 1 / 2}^{(i)}+$ $\lambda_{\theta, 1 / 2}^{(i)} x_{i, t}+\gamma_{\theta, 1 / 2}^{(i)} f p c_{t-1}$

\begin{tabular}{|c|c|c|c|c|c|c|c|c|c|c|}
\hline $\mathrm{COEF}$ & $5 \mathrm{P}$ & MED & $95 \mathrm{P}$ & IQR & PS & $5 \mathrm{P}$ & MED & $95 \mathrm{P}$ & IQR & PS \\
\hline & \multicolumn{5}{|c|}{$\theta=\tau=0.01, h=0.10$} & \multicolumn{5}{|c|}{$\theta=\tau=0.05, h=0.10$} \\
\hline$\delta_{\theta, \tau}$ & -0.050 & -0.029 & -0.015 & 0.015 & 91.262 & -0.031 & -0.019 & -0.010 & 0.008 & 94.175 \\
\hline$\delta_{\theta, 1 / 2}$ & -0.041 & -0.028 & -0.017 & 0.012 & 97.864 & -0.025 & -0.017 & -0.011 & 0.006 & 98.447 \\
\hline$\lambda_{\theta, \tau}$ & -0.213 & 0.218 & 0.980 & 0.518 & 24.660 & -0.089 & 0.205 & 0.884 & 0.471 & 35.728 \\
\hline$\lambda_{\theta, 1 / 2}$ & -0.470 & 0.349 & 1.135 & 0.588 & 32.427 & -0.199 & 0.354 & 0.910 & 0.453 & 46.019 \\
\hline $100 \times \gamma_{\theta, \tau}$ & -0.392 & -0.193 & 0.002 & 0.155 & 72.816 & -0.258 & -0.146 & -0.042 & 0.080 & 84.563 \\
\hline \multirow[t]{2}{*}{$100 \times \gamma_{\theta, 1 / 2}$} & -0.302 & -0.176 & -0.037 & 0.102 & 84.660 & -0.182 & -0.113 & -0.058 & 0.058 & 89.709 \\
\hline & \multicolumn{5}{|c|}{$\theta=\tau=0.01, h=0.15$} & \multicolumn{5}{|c|}{$\theta=\tau=0.05, h=0.15$} \\
\hline$\delta_{\theta, \tau}$ & -0.049 & -0.029 & -0.016 & 0.014 & 95.049 & -0.030 & -0.018 & -0.010 & 0.007 & 95.437 \\
\hline$\delta_{\theta, 1 / 2}$ & -0.041 & -0.028 & -0.019 & 0.011 & 99.223 & -0.026 & -0.018 & -0.012 & 0.007 & 99.612 \\
\hline$\lambda_{\theta, \tau}$ & -0.199 & 0.248 & 1.025 & 0.559 & 30.874 & -0.081 & 0.212 & 0.902 & 0.471 & 40.485 \\
\hline$\lambda_{\theta, 1 / 2}$ & -0.163 & 0.214 & 0.788 & 0.387 & 41.650 & -0.047 & 0.231 & 0.731 & 0.366 & 55.340 \\
\hline $100 \times \gamma_{\theta, \tau}$ & -0.375 & -0.194 & -0.013 & 0.143 & 77.573 & -0.242 & -0.143 & -0.061 & 0.076 & 88.932 \\
\hline \multirow[t]{2}{*}{$100 \times \gamma_{\theta, 1 / 2}$} & -0.300 & -0.180 & -0.045 & 0.099 & 86.214 & -0.186 & -0.115 & -0.066 & 0.059 & 92.136 \\
\hline & \multicolumn{5}{|c|}{$\theta=\tau=0.01, h=0.20$} & \multicolumn{5}{|c|}{$\theta=\tau=0.05, h=0.20$} \\
\hline$\delta_{\theta, \tau}$ & -0.048 & -0.029 & -0.017 & 0.013 & 97.087 & -0.029 & -0.018 & -0.011 & 0.007 & 96.505 \\
\hline$\delta_{\theta, 1 / 2}$ & -0.041 & -0.029 & -0.020 & 0.011 & 99.515 & -0.026 & -0.018 & -0.012 & 0.007 & 99.612 \\
\hline$\lambda_{\theta, \tau}$ & -0.168 & 0.260 & 0.951 & 0.538 & 34.078 & -0.056 & 0.227 & 0.890 & 0.475 & 44.078 \\
\hline$\lambda_{\theta, 1 / 2}$ & -0.093 & 0.154 & 0.624 & 0.308 & 47.184 & -0.015 & 0.160 & 0.650 & 0.331 & 60.388 \\
\hline $100 \times \gamma_{\theta, \tau}$ & -0.357 & -0.197 & -0.039 & 0.132 & 83.786 & -0.231 & -0.142 & -0.068 & 0.071 & 90.583 \\
\hline $100 \times \gamma_{\theta, 1 / 2}$ & -0.299 & -0.186 & -0.059 & 0.092 & 88.252 & -0.190 & -0.118 & -0.071 & 0.060 & 94.660 \\
\hline
\end{tabular}

The table reports the summary statistics of the QL-CoVaR's parameters estimated for the $N$ financial companies included in our dataset. We estimated the conditional quantiles for two quantile levels $(\theta)$ and three bandwidth levels $(h)$. In each panel, from left to right, we report the following descriptive statistics of the coefficients: the 5 -th percentile (5P), the median (MED), the 95-th percentile (95P), the interquartile range (IQR) and the percentage of times, over the cross-section, in which they are statistically significant at the $5 \%$ confidence level (PS). 
risk of the system. Therefore, a very bad or a very good day symmetrically increases the probability of observing greater losses in the next day, consistent with the hypotheses laid down by Engle and Manganelli (2004). When analyzing the coefficients associated with this symmetric innovation, we first note that $\widehat{\psi}_{2,0.01}^{(i)}$ is slightly more volatile than $\widehat{\psi}_{2,0.05}^{(i)}$, as the interquartile range of the former (the latter) is equal to $0.203(0.133)$. $\widehat{\psi}_{2, \theta}^{(i)}$ records a lower percentage of times in which it is statistically significant when compared to the other CaViaR's parameter, that is, $\widehat{\psi}_{1, \theta}^{(i)}$. Moving to the loadings of both the individual company's returns and the first principal component of the control variables, we observe that the statistics of $\hat{\lambda}_{\theta}^{(i)}$ and of $\widehat{\gamma}_{\theta}^{(i)}$ slightly change when switching from the CoVaR to the CoCaViaR. The individual companies have, on average, a positive impact on the financial system in the CoCaViaR model. $f p c_{t-1}$ is statistically relevant in a large fraction of cases. However, we observe a contraction in the estimates for both $\widehat{\lambda}_{\theta}^{(i)}$ and $\widehat{\gamma}_{\theta}^{(i)}$ when moving from the CoVaR to the CoCaViaR. This is due to the presence of the CaViaR's components in the latter model.

Note that the CoCaViaR's parameters are estimated from a quantile regression model that includes two regressors observed in $t-1:\left|y_{t-1}\right|$ and $f p c_{t-1}$. Both variables filter the contemporaneous relations between the financial system and the $i$-th company, by including the lagged impact of the system's returns and of state variables, respectively. It is interesting to analyze how the lagged interconnections between $\left|y_{t-1}\right|$ and $f p c_{t-1}$ in $t-1$ impact on the contemporaneous relations between the system's quantiles and the $i$-th company. For this purpose, we implement a robustness check in which we exclude either $\left|y_{t-1}\right|$ or $f p c_{t-1}$ from the CoCaViaR model. We report the results in Table S4. We first note that the coefficients related to the CaViaR components- $\psi_{1, \theta}$ and $\psi_{2, \theta}$, especially $\psi_{1, \theta}$ - take greater absolute values, on average, when excluding $f p c_{t-1}$ (see panel (a) of Table S4). Therefore, including additional lagged variables into the CoCaViaR model reduces the relevance of the CaViaR components. We observe the same phenomenon when excluding $\left|y_{t-1}\right|$ : the impact of $f p c_{t-1}$ increases, albeit to a lesser extent with respect to the previous case. However, $\lambda_{\theta}$ does not exhibit relevant changes when excluding either $\left|y_{t-1}\right|$ or $f p c_{t-1}$. Finally, it is interesting to observe that excluding one regressor from the model (either $\left|y_{t-1}\right|$ or $f p c_{t-1}$ ) reduces the interquartile range of the coefficients.

In contrast to the models analyzed above, the estimation process behind the QL-CoVaR (and thus the QL-CoCaViaR) depends on two additional parameters: a second quantile $\tau$ (we now restrict the attention on the neighborhood of the $\tau$-th quantile of $x_{i, t}$ ) and a bandwidth $h$ (that calibrates the weight of the kernel function). Table S5 reports the statistics of the QL-CoVaR's coefficients, where we condition the estimates to the distress and to the median state of an individual company. As before, the average impact exerted by the companies to both $Q L-C o V a R_{\tau}^{(i)}$ and $Q L-C o V a R_{1 / 2}^{(i)}$ is positive, but greater with respect to the standard CoVaR (the medians of both $\hat{\lambda}_{\theta, \tau}^{(i)}$ and $\widehat{\lambda}_{\theta, 0.5}^{(i)}$ are greater than the median of $\widehat{\lambda}_{\theta}^{(i)}$ ). Therefore, the relationships between the system and the companies become stronger when focusing on particular regions of the $x_{i, t}$ 's support. On average, we observe larger values for $\hat{\lambda}_{\theta, \tau}^{(i)}$ at $\theta=0.01$ than at $\theta=0.05$, whereas the opposite holds for $\widehat{\lambda}_{\theta, 0.5}^{(i)} \cdot \widehat{\lambda}_{\theta, \tau}^{(i)}$ measures the relation between 
$x_{i, t}$ and $y_{t}$, when the companies and the system simultaneously lie in the left tails of their distributions. The fact that $\widehat{\lambda}_{\theta, \tau}^{(i)}$ increases as $\theta$ and $\tau$ simultaneously decrease means that the co-movements between the financial system and the companies become stronger when moving leftwards along the left tails of their distributions. As a result, the risk of contagion increases by accentuating the distress degree in the connections between the financial system and the individual companies. The relevance of the co-movements between the financial system and the individual companies emerges also in their median state. In fact, $\widehat{\lambda}_{\theta, 0.5}^{(i)}$ increases as the system moves rightwards from $\theta=0.01$ to $\theta=0.05$, reducing the gap between the median state of the conditioning company and the distress state of the system.

Table S6: Estimation of $Q_{\theta, \tau}^{(i)}\left(y_{t}\right)=\delta_{\theta, \tau}^{(i)}+\psi_{1, \theta, \tau}^{(i)} Q_{\theta, \tau}^{(i)}\left(y_{t-1}\right)+\psi_{2, \theta, \tau}^{(i)}\left|y_{t-1}\right|+\lambda_{\theta, \tau}^{(i)} x_{i, t}+\gamma_{\theta, \tau}^{(i)} f p c_{t-1}$ and $Q_{\theta, 1 / 2}^{(i)}\left(y_{t}\right)=\delta_{\theta, 1 / 2}^{(i)}+\psi_{1, \theta, 1 / 2}^{(i)} Q_{\theta, 1 / 2}^{(i)}\left(y_{t-1}\right)+\psi_{2, \theta, 1 / 2}^{(i)}\left|y_{t-1}\right|+\lambda_{\theta, 1 / 2}^{(i)} x_{i, t}+\gamma_{\theta, 1 / 2}^{(i)} f p c_{t-1}$

\begin{tabular}{|c|c|c|c|c|c|c|c|c|c|c|}
\hline $\mathrm{COEF}$ & $5 \mathrm{P}$ & MED & $95 \mathrm{P}$ & IQR & PS & $5 \mathrm{P}$ & MED & $95 \mathrm{P}$ & IQR & PS \\
\hline & \multicolumn{5}{|c|}{$\theta=\tau=0.01, h=0.10$} & \multicolumn{5}{|c|}{$\theta=\tau=0.05, h=0.10$} \\
\hline$\delta_{\theta, \tau}$ & -0.059 & -0.020 & -0.001 & 0.024 & 61.456 & -0.033 & -0.013 & 0.000 & 0.014 & 57.476 \\
\hline$\delta_{\theta, 1 / 2}$ & -0.041 & -0.011 & 0.000 & 0.020 & 67.184 & -0.026 & -0.010 & 0.000 & 0.014 & 56.602 \\
\hline$\psi_{1, \theta, \tau}$ & -0.698 & 0.181 & 0.919 & 0.829 & 45.534 & -0.739 & 0.265 & 0.932 & 0.782 & 48.350 \\
\hline$\psi_{1, \theta, 1 / 2}$ & -0.582 & 0.491 & 0.957 & 0.842 & 65.049 & -0.67 & 0.254 & 0.949 & 0.849 & 55.340 \\
\hline$\psi_{2, \theta, \tau}$ & -0.602 & -0.062 & 0.568 & 0.449 & 26.505 & -0.355 & -0.073 & 0.393 & 0.282 & 25.437 \\
\hline$\psi_{2, \theta, 1 / 2}$ & -0.570 & -0.171 & 0.278 & 0.247 & 50.388 & -0.318 & -0.112 & 0.216 & 0.146 & 38.155 \\
\hline$\lambda_{\theta, \tau}$ & -0.287 & 0.147 & 0.843 & 0.458 & 54.466 & -0.125 & 0.121 & 0.855 & 0.437 & 53.01 \\
\hline$\lambda_{\theta, 1 / 2}$ & -0.250 & 0.086 & 0.906 & 0.379 & 51.942 & -0.112 & 0.091 & 0.853 & 0.454 & 46.796 \\
\hline $100 \times \gamma_{\theta, \tau}$ & -0.410 & -0.113 & 0.004 & 0.173 & 52.913 & -0.232 & -0.089 & 0.001 & 0.117 & 52.718 \\
\hline \multirow[t]{2}{*}{$100 \times \gamma_{\theta, 1 / 2}$} & -0.233 & -0.049 & 0.004 & 0.120 & 57.670 & -0.177 & -0.057 & 0.001 & 0.091 & 51.456 \\
\hline & \multicolumn{5}{|c|}{$\theta=\tau=0.01, h=0.15$} & \multicolumn{5}{|c|}{$\theta=\tau=0.05, h=0.15$} \\
\hline$\delta_{\theta, \tau}$ & -0.054 & -0.021 & -0.001 & 0.023 & 67.184 & -0.031 & -0.013 & 0.000 & 0.013 & 61.068 \\
\hline$\delta_{\theta, 1 / 2}$ & -0.040 & -0.012 & 0.00 & 0.021 & 73.592 & -0.027 & -0.011 & 0.000 & 0.014 & 65.146 \\
\hline$\psi_{1, \theta, \tau}$ & -0.619 & 0.136 & 0.918 & 0.761 & 45.049 & -0.746 & 0.221 & 0.927 & 0.754 & 47.670 \\
\hline$\psi_{1, \theta, 1 / 2}$ & -0.630 & 0.467 & 0.949 & 0.840 & 66.019 & -0.662 & 0.220 & 0.937 & 0.830 & 55.437 \\
\hline$\psi_{2, \theta, \tau}$ & -0.533 & -0.060 & 0.479 & 0.386 & 27.184 & -0.317 & -0.085 & 0.323 & 0.237 & 29.029 \\
\hline$\psi_{2, \theta, 1 / 2}$ & -0.519 & -0.172 & 0.234 & 0.219 & 54.660 & -0.281 & -0.116 & 0.156 & 0.129 & 41.748 \\
\hline$\lambda_{\theta, \tau}$ & -0.218 & 0.174 & 0.902 & 0.496 & 57.573 & -0.096 & 0.131 & 0.855 & 0.448 & 54.66 \\
\hline$\lambda_{\theta, 1 / 2}$ & -0.125 & 0.091 & 0.711 & 0.284 & 64.660 & -0.047 & 0.109 & 0.722 & 0.396 & 57.573 \\
\hline $100 \times \gamma_{\theta, \tau}$ & -0.402 & -0.126 & 0.002 & 0.173 & 60.485 & -0.220 & -0.091 & 0.001 & 0.113 & 57.573 \\
\hline \multirow{2}{*}{$100 \times \gamma_{\theta, 1 / 2}$} & -0.227 & -0.053 & 0.003 & 0.128 & 63.592 & -0.163 & -0.068 & 0.000 & 0.089 & 60.777 \\
\hline & \multicolumn{5}{|c|}{$\theta=\tau=0.01, h=0.20$} & \multicolumn{5}{|c|}{$\theta=\tau=0.05, h=0.20$} \\
\hline$\delta_{\theta, \tau}$ & -0.049 & -0.022 & -0.001 & 0.022 & 75.049 & -0.03 & -0.013 & 0.000 & 0.013 & 66.602 \\
\hline$\delta_{\theta, 1 / 2}$ & -0.042 & -0.014 & -0.001 & 0.022 & 80.971 & -0.027 & -0.012 & 0.000 & 0.014 & 72.913 \\
\hline$\psi_{1, \theta, \tau}$ & -0.601 & 0.133 & 0.923 & 0.721 & 46.117 & -0.687 & 0.176 & 0.921 & 0.745 & 46.699 \\
\hline$\psi_{1, \theta, 1 / 2}$ & -0.605 & 0.401 & 0.939 & 0.870 & 66.019 & -0.660 & 0.186 & 0.927 & 0.823 & 55.437 \\
\hline$\psi_{2, \theta, \tau}$ & -0.510 & -0.074 & 0.384 & 0.333 & 32.816 & -0.278 & -0.087 & 0.283 & 0.204 & 32.330 \\
\hline$\psi_{2, \theta, 1 / 2}$ & -0.463 & -0.175 & 0.198 & 0.205 & 55.437 & -0.254 & -0.122 & 0.117 & 0.121 & 42.816 \\
\hline$\lambda_{\theta, \tau}$ & -0.194 & 0.185 & 0.886 & 0.525 & 64.757 & -0.072 & 0.139 & 0.85 & 0.467 & 59.806 \\
\hline$\lambda_{\theta, 1 / 2}$ & -0.068 & 0.096 & 0.600 & 0.263 & 73.883 & -0.022 & 0.103 & 0.652 & 0.351 & 65.243 \\
\hline $100 \times \gamma_{\theta, \tau}$ & -0.343 & -0.135 & 0.002 & 0.162 & 68.252 & -0.207 & -0.089 & 0.000 & 0.105 & 62.233 \\
\hline $100 \times \gamma_{\theta, 1 / 2}$ & -0.230 & -0.067 & 0.001 & 0.138 & 70.874 & -0.161 & -0.071 & -0.001 & 0.090 & 69.029 \\
\hline
\end{tabular}

The table reports the summary statistics of the QL-CoCaViaR's parameters estimated for the $N$ financial companies included in our dataset. We estimated the conditional quantiles for two quantile levels $(\theta)$ and three bandwidth levels $(h)$. In each panel, from left to right, we report the following descriptive statistics of the coefficients: the 5-th percentile $(5 \mathrm{P})$, the median (MED), the 95-th percentile (95P), the interquartile range (IQR) and the percentage of times, over the cross-section, in which they are statistically significant at the $5 \%$ confidence level (PS).

Finally, the statistics of the QL-CoCaViaR's coefficients are given in Table S6. On average, $\widehat{\lambda}_{\theta, \tau}^{(i)}$ 
takes greater values at $\theta=0.01$ than at $\theta=0.05$ and the opposite holds for $\widehat{\lambda}_{\theta, 0.5}^{(i)}$, highlighting the stronger co-movements between the system and the companies when they simultaneously lie in a state of strong distress. Switching from the QL-CoVaR to the QL-CoCaViaR, the inclusion of the CaViaR components absorbs, in part, the impact of the companies on the system: the medians of $\hat{\lambda}_{\theta, \tau}^{(i)}$ and $\widehat{\lambda}_{\theta, 0.5}^{(i)}$ are lower than the ones observed in the case of the QL-CoVaR. The effects of changing the value of $h$ are moderate in terms of median, interquartile range, 5-th and 95-th percentile of the estimated parameters. Changing the value of $h$ does not imply relevant consequences in terms of times in which the model's coefficients are statistically significant over the cross-section. As in the case of the CoCaViaR, the values of $\psi_{1, \theta, \tau}^{(i)}$ and $\psi_{1, \theta, 1 / 2}^{(i)}$ are positive, on average, but lower than the ones typically observed in the standard CaViaR model. It is interesting to observe that the median of $\psi_{1, \theta, \tau}^{(i)}$ is lower at $\theta=\tau=0.01$ than at $\theta=\tau=0.05$, in contrast to what occurs in the case of the CoCaViaR. In contrast, the median of $\psi_{1, \theta, 0.5}^{(i)}$ is greater at $\theta=0.01$ than at $\theta=0.05$. This phenomenon might be due to the fact that the persistence of the $y_{t}$ 's quantiles is affected by the relations between the company and the system. In fact, as described above, the co-movements between the system and the companies are stronger as $\theta$ and $\tau$ simultaneously take lower values and this absorbs, in part, the persistence of $Q_{\theta, \tau}^{(i)}\left(y_{t}\right)$. In contrast, the $y_{t}$ 's quantiles are more persistent when the co-movements between $y_{t}$ and $x_{i, t}$ are weaker, that is, when the system is in distress, whereas the $i$-th company is in its median state. As a result, on average, $\psi_{1,0.01,0.01}^{(i)}$ is less than half $\psi_{1,0.01,0.5}^{(i)}$, whereas the differences between $\psi_{1,0.05,0.05}^{(i)}$ and $\psi_{1,0.05,0.5}^{(i)}$ are almost imperceptible. Similar to what we observe for the QL-CoVaR, $\left|y_{t-1}\right|$ has a negative impact on $Q_{\theta, \tau}^{(i)}\left(y_{t}\right) . \psi_{2, \theta, \tau}^{(i)}$ is greater, in absolute value, at $\theta=\tau=0.05$ than at $\theta=\tau=0.01$, whereas the opposite holds for $\psi_{2, \theta, 0.5}^{(i)}$. Then, also the impact of the lagged system's returns is affected by the relationships between $y_{t}$ and $x_{i, t}$, according to the normal or distress state in which they are. Furthermore, changes in the values of $h$ have slight effects on the statistics of $\psi_{1, \theta, \tau}^{(i)}, \psi_{1, \theta, 0.5}^{(i)}, \psi_{2, \theta, \tau}^{(i)}$ and $\psi_{2, \theta, 0.5}^{(i)}$. Finally, in contrast to the QL-CoVaR model, we note that the percentage of companies for which the QL-CoCaViaR's coefficients are statistically significant at the $5 \%$ confidence level tends to be greater at the quantiles levels $(\theta, 1 / 2)$ than $(\theta, \tau)$ and is almost always a positive function of $h$.

\section{S4 Additional results}

\section{S4.1 Backtesting systemic risk measures}

We compute the average $\Delta \mathrm{CoVaR}$ for each company for which we have no missing values in the pre-crisis period (the number of which is equal to 390). We then sort these companies (in ascending order) according to these average values, from the company with rank 1 (which has, on average, the greatest impact on the systemic risk prior to the subprime crisis) to the company with rank 390 (which has the lowest impact). We implement the same procedure for the other $\Delta$ Risk measures, obtaining a different classification for each of them. From each classification, we select the companies with rank 
lower or equal to $R^{\star}$, that constitute the group of the $R^{\star}$ companies having the greatest impact on the systemic risk. We use three different values of $R^{\star}$ — that is, 10, 50 and 100 - and report the percentage of banks among the most relevant $R^{\star}$ companies prior to the subprime crisis in Table S7. It is possible to see from Table S7 that banks constitute the majority of the top-ranked institutions. These results are in line with Billio et al. (2012) and Girardi and Ergun (2013), which found that, prior to the crisis, banks are among the highest contributors to systemic risk, whereas insurers are among the lowest. Notably, we can see from Table S7 that this phenomenon becomes more evident when using the $\Delta \mathrm{QL}-\mathrm{CoCaViaR}$.

Table S7: The relevance of banks prior to the subprime crisis

\begin{tabular}{c|cccc}
\hline$R^{\star}$ & $\Delta$ CoVaR & $\Delta$ CoCaViaR & $\Delta$ QL-CoVaR & $\Delta$ QL-CoCaViaR \\
\hline 10 & $70 \%$ & $80 \%$ & $80 \%$ & $80 \%$ \\
50 & $66 \%$ & $78 \%$ & $78 \%$ & $80 \%$ \\
100 & $69 \%$ & $73 \%$ & $70 \%$ & $76 \%$ \\
\hline
\end{tabular}

The table reports, for each $\Delta$ Risk measure, the percentages of banks among the $R^{\star}$ companies that have, on average, the greatest impact on the systemic risk during the period June 1, 2006-May 31, 2007.

\section{S4.2 Systemic risk and quantile location}

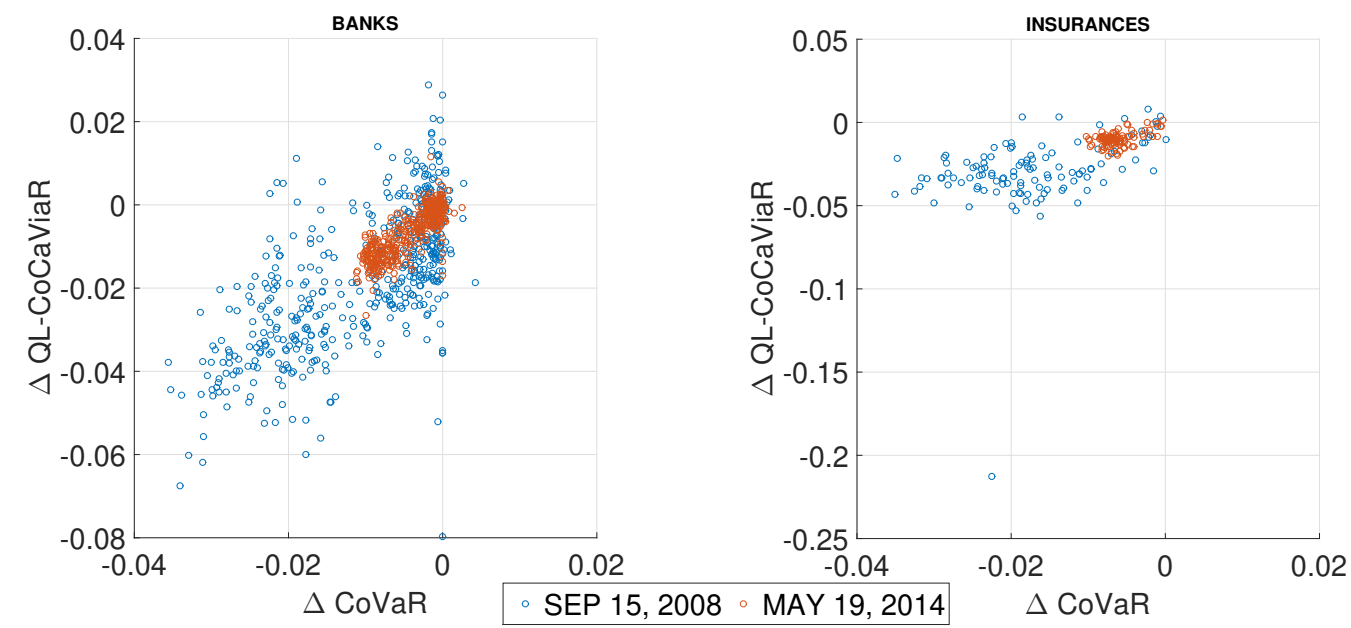

Figure S2: Scatter plots of $\Delta$ CoVaR and $\Delta$ QL-CoCaViaR on two specific days: 15/09/2008 and 19/05/2014. The risk measures are computed at $\theta=\tau=0.01$ and $h=0.15$.

We compare the $\Delta \mathrm{CoVaR}$ with the $\Delta \mathrm{QL}-\mathrm{CoCaViaR}$ in a stressed (September 15,2008 ) and in a quiet day (May 19, 2014). For simplicity, we compare here the $\Delta$ CoVaR with the $\Delta$ QL-CoCaViaR only. We stress that, if the differences between the two measures on the two different days were due to a volatility effect only (without any additional element coming from the covariates and the conditional quantile model structure), we would have not noted an increase in the scatter plot dispersion. In fact, the scatter plots would have displayed similar changes of the volatility (assuming all other elements play no role), providing a change in the location only, and not in the scale. However, we observe a marked increase of both the location of the scatter center and the dispersion in Figure S2. This is 
something that we cannot simply attribute to a change in the market risk over the two specific days. In addition, the relations between the $\Delta \mathrm{CoVaR}$ and the $\Delta \mathrm{QL}-\mathrm{CoCaViaR}$ seem much stronger when the market is in a low volatility state. We link this evidence to the possible structural changes in the relations between the financial system, the individual companies and their corresponding quantiles, which might be observed during market turmoils. Moving from the $\Delta$ CoVaR to the $\Delta \mathrm{QL}-\mathrm{CoCaViaR}$ does not simply induce a linear shift of the systemic risk measures but accounts in a more proper way for the relations between the companies and the system when focusing on extreme market conditions.

\section{S5 Additional figures}

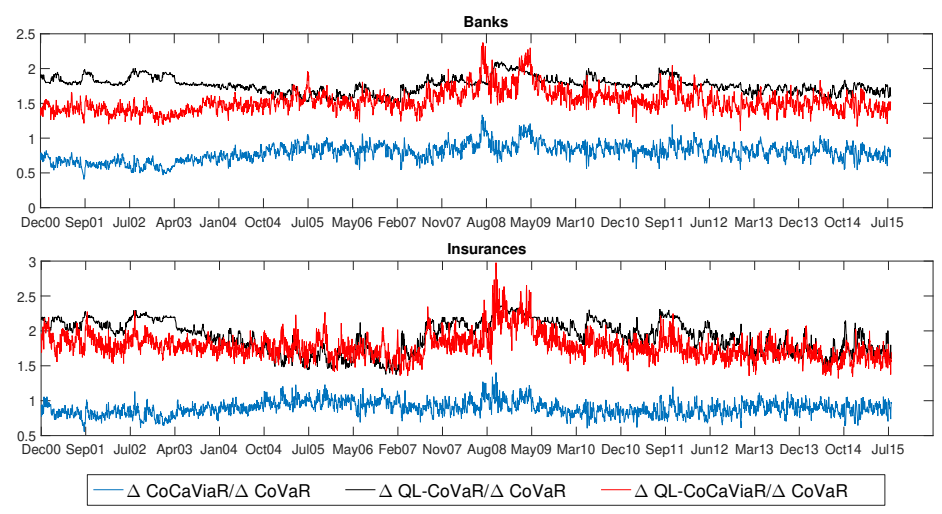

Figure S3: For each day in the period December 2000 - July 2015, the figure displays the cross-sectional medians of the ratios $\Delta \mathrm{CoCaViaR} / \Delta \mathrm{CoVaR}, \Delta \mathrm{QL}-\mathrm{CoVaR} / \Delta \mathrm{CoVaR}$ and $\Delta \mathrm{QL}-\mathrm{CoCaViaR} / \Delta \mathrm{CoVaR}$, distinguishing banks from insurances. The risk measures are computed at $\theta=\tau=0.01$ and $h=0.15$. The results obtained at $\theta=\tau=0.05$ and $h=\{0.10,0.20\}$ are qualitative similar and available upon request.
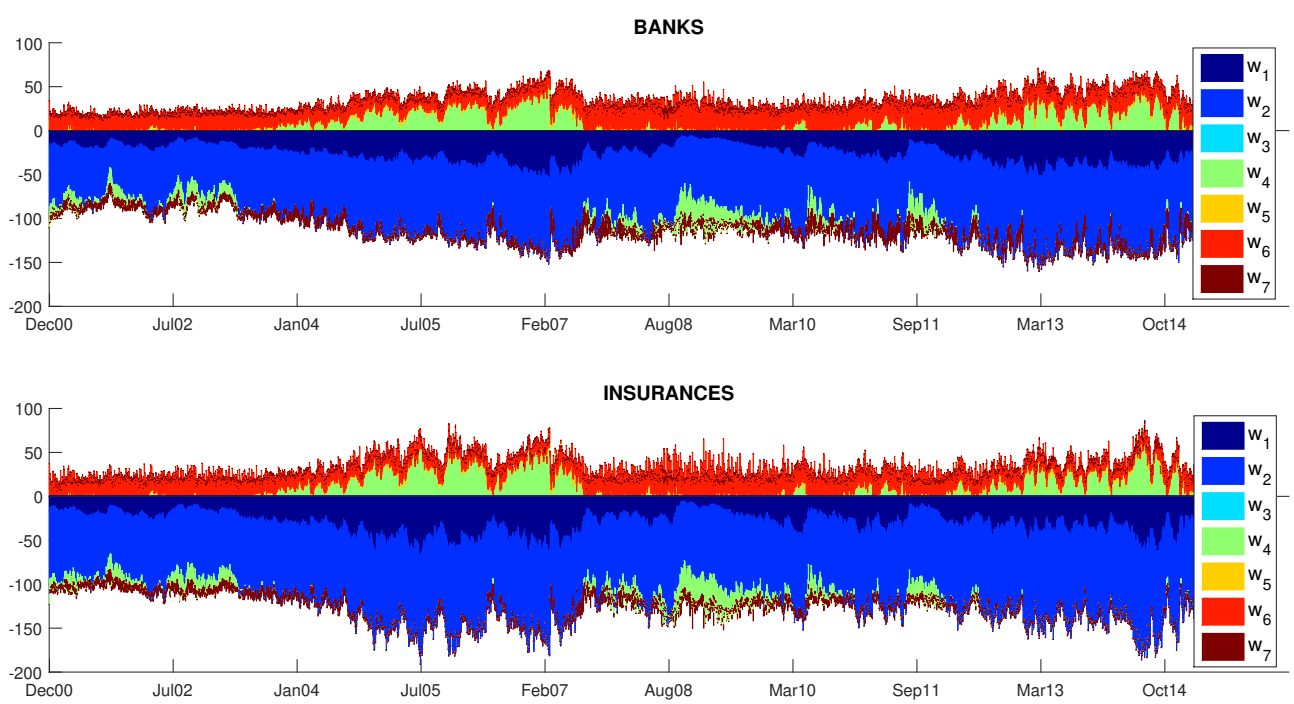

Figure S4: For each day from December 2000 to July 2015, the figure displays the cross-sectional medians (\%) of the $w_{i, \tau}$ ratios, for $i=1,2, \ldots 7$, distinguishing banks from insurances. The underlying risk measures are computed at $\theta=\tau=0.01$ and $h=0.15$. 

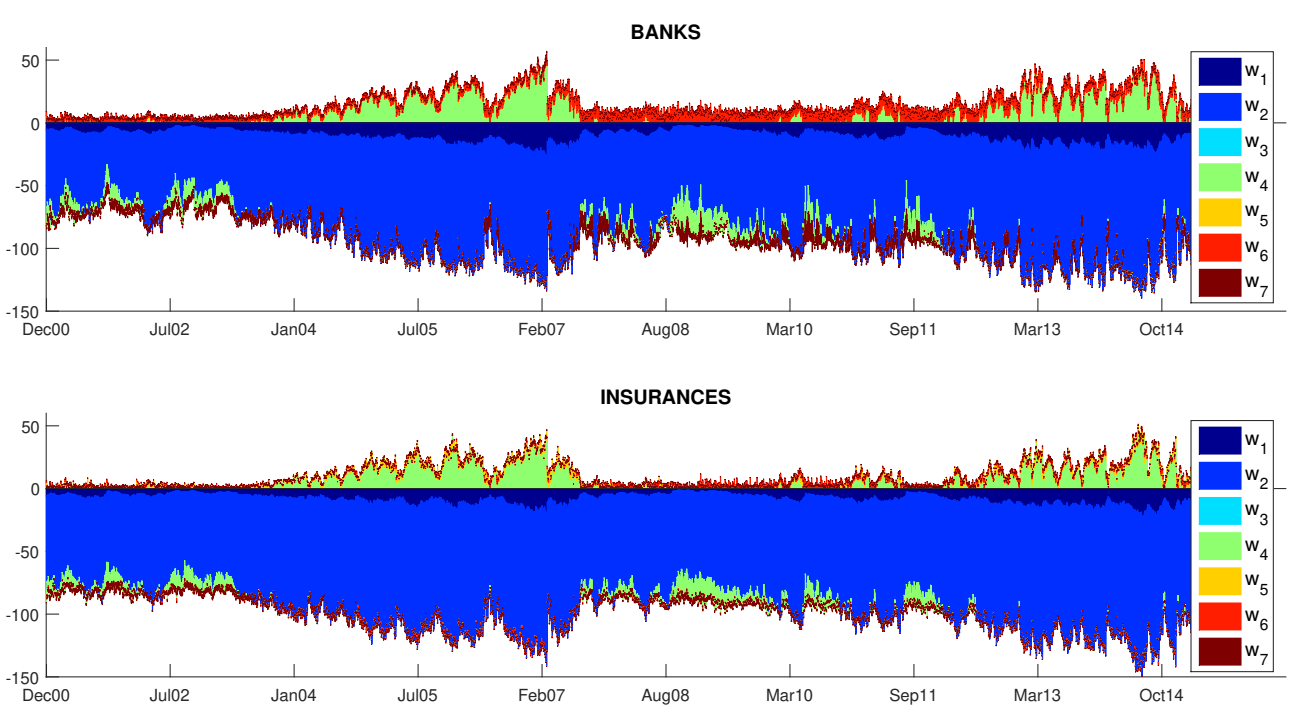

Figure S5: For each day from December 2000 to July 2015, the figure displays the cross-sectional medians (\%) of the $w_{i, \tau}$ ratios, for $i=1,2, \ldots 7$, distinguishing banks from insurances. The underlying risk measures are computed at $\theta=\tau=0.05$ and $h=0.15$.

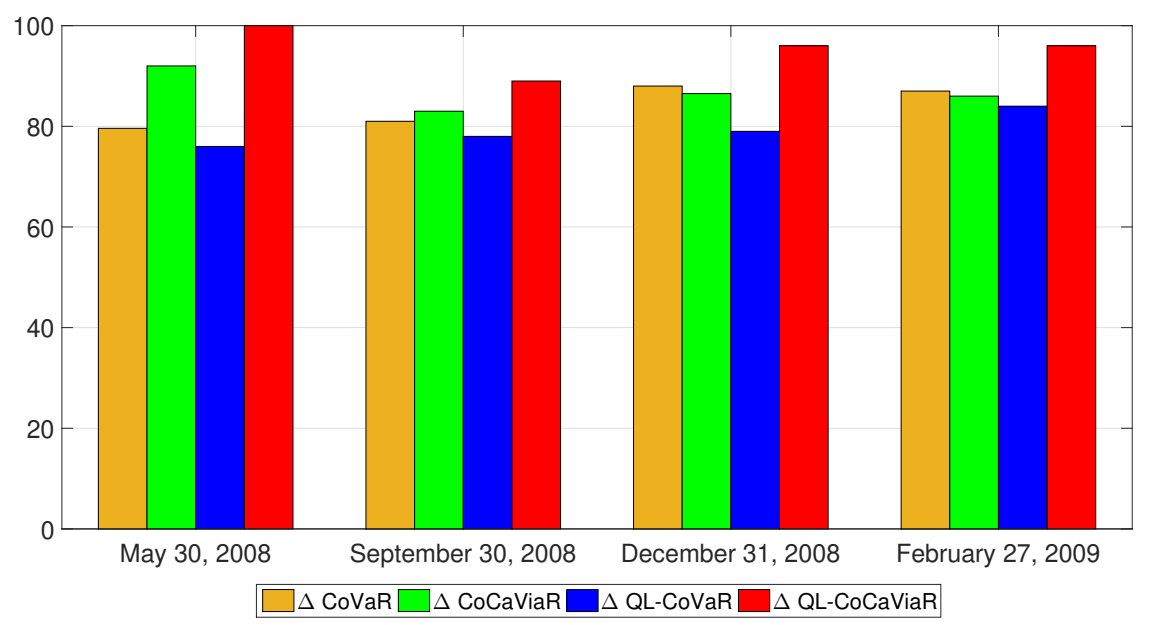

Figure S6: $C R$ ratio (\%) generated by the competitive $\Delta$ Risk measures $-\Delta$ CoVaR, $\Delta$ CoCaViaR, $\Delta$ QL-CoVaR and $\triangle \mathrm{QL}-\mathrm{CoCaViaR}$ - at different concluding dates of the crisis period, that is, May 30, 2008, September 30, 2008, December 31, 2008 and February 27, 2009. Here, we compute the $C R$ ratio as the proportion of companies selected by the LASSO model that, at the same time, are also classified in the first three groups of the ex-ante ranking. 